\title{
Desarrollo de la capacidad para la regulación en salud en México
}

\author{
Carlos Santos-Burgoa, ${ }^{1}$ Manuel Urbina-Fuentes, ${ }^{2}$ Juan Ángel Rivera-Dommarco, ${ }^{3}$ Martha Hijar, ${ }^{4}$ \\ Julio Salvador Sánchez-Tépoz, ${ }^{2}$ Juan Carlos Gallaga-Solórzano, ${ }^{5}$ Rocío del Carmen Alatorre Eden-Wynter, \\ Armida Zúñiga-Estrada, ${ }^{5}$ Ricardo García-Sarubbi, ${ }^{6}$ Claudia Gabriela García-Chávez ${ }^{7}$ y Ana Carolina Ariza ${ }^{8}$ \\ ${ }^{1}$ The George Washington University, Milken Institute School of Public Health, Washington DC, EE. UU.; ${ }^{2}$ Academia Nacional de Medicina, Comité \\ Permanente para el Estudio de los Determinantes Sociales de la Salud, Ciudad de México, México; ${ }^{3}$ Secretaría de Salud, Instituto Nacional de \\ Salud Pública, Dirección General, Ciudad de México, México; ${ }^{4}$ Fundación Entornos, Morelos, México; ${ }^{5}$ Comisión Federal para la Protección contra \\ Riesgos Sanitarios, Ciudad de México, México; ${ }^{6}$ Secretaría de Salud, Secretariado Técnico del Consejo Nacional para la Prevención de Accidentes, \\ Ciudad de México, México; ' Secretaría de Salud, Instituto Nacional de Salud Pública, Centro de Investigación en Nutrición y Salud, Ciudad de \\ México, México; ${ }^{8}$ Consejo Nacional de Ciencia y Tecnología, Ciudad de México, México
}

\begin{abstract}
Resumen
La Academia Nacional de Medicina es un espacio esencial para discutir la ciencia de la regulación en salud y posicionar su impacto en la salud y la economía. Enmarcada dentro de la función rectora de la autoridad sanitaria, la regulación en salud es la acción de proteger a la población de los peligros sanitarios involuntarios contra los cuales el individuo no puede protegerse; es una función esencial de la salud pública, componente institucional del sistema de salud y, por ende, vinculada a sus reformas y a la cobertura universal. La regulación tiene sustento en un cuerpo teórico epidemiológico, organizacional, legal, sociológico y económico. Tiene un cuerpo metodológico que sustenta su proceso en el análisis de riesgos y se traduce en normas, implementaciones, cumplimiento, monitoreo y evaluación de la regulación. Tiene una arquitectura profesional, financiera, organizacional, legal y de gobernanza. Dada su acción universal tiene un impacto generalizado en la población y un sustancial efecto económico, influyendo en al menos $17 \%$ del comercio internacional regional. La salud a través de sus autoridades regulatorias debe ser parte del dialogo comercial internacional.
\end{abstract}

PALABRAS CLAVE: Regulación. Sistema de salud. Desarrollo. Globalización. Enfermedades no transmisibles. Gobernanza.

\begin{abstract}
The National Academy of Medicine is an essential space to discuss regulatory science in health, and to position its impact on health and economy. Framed within the stewardship role of the health authority, health regulation is the action of protecting the population against involuntary health hazards against which the individual cannot protect him/herself. It is an essential function of public health, an institutional component of the health system and, therefore, linked to its reforms and to universal coverage. Regulation has its support on an epidemiological, organizational, legal, sociological and economic theoretical body. It has a methodological body that supports its regulatory process based on risk analysis and that is translated into regulations, implementations, compliance, monitoring and evaluation of the regulation. It has a professional, financial, organizational, legal and governance architecture. Given its universal action, it has a widespread impact on the population and a substantial economic effect, influencing on at least $17 \%$ of regional international trade. Health through its regulatory authorities should be an early part of international trade discussions.
\end{abstract}

KEY WORDS: Regulation. Health system. Development. Globalization. Noncommunicable diseases. Governance.

Correspondencia:

Carlos Santos-Burgoa

E-mail: csantosburgoa@gwu.edu
Fecha de recepción: 21-06-2017

Fecha de aceptación: 18-04-2018

DOI://dx.doi.org/10.24875/GMM.18004351
Gac Med Mex. 2018;154:368-390

Disponible en PubMed www.anmm.org. $\mathrm{mx}$ 


\title{
Marco general de la regulación en salud en el contexto político global
}

\author{
Carlos Santos-Burgoa
}

\section{Introducción}

La Academia Nacional de Medicina es un espacio esencial para la discusión sobre la ciencia de la regulación en salud, dado su carácter de órgano consultivo independiente del Estado mexicano, en relación con las políticas públicas de salud. Es necesario posicionar la función regulatoria en salud pública, ${ }^{1}$ por la magnitud de su impacto, y reconocer el beneficio de avanzar en la capacidad técnica, científica, gobernanza y operativa en este aspecto.

Dentro de la función rectora de la autoridad sanitaria, ${ }^{2}$ la regulación en salud consiste en la acción de proteger a la población contra los peligros sanitarios involuntarios fuera del alcance de los individuos, acción que se complementa con la promoción de la salud y la prevención clínica. Conlleva la evaluación sistemática de los riesgos, el ordenamiento saludable de productos, servicios y entornos, el impulso a los bienes comunes, la responsabilidad social y el mejor desarrollo de las personas y sus comunidades, con sustento en bases científicas. Es una función esencial del sistema de salud, íntimamente vinculada al desarrollo económico y social, ejercida por la autoridad sanitaria con la participación de profesionales de la salud pública. ${ }^{3}$

\section{Base conceptual de la regulación en salud}

Para el caso de México, la reforma en salud de 2003 consideró la protección contra riesgos a la salud junto con la calidad de la atención y la protección financiera. ${ }^{4}$

La regulación en salud se fundamenta en un cuerpo teórico que se remonta a la epidemiología y al control sanitario implementado formalmente por John Snow, quien sustentó en datos científicos que la autoridad de salud debía hacer actuar a las autoridades locales para suprimir la fuente de agua transmisora del cólera en Londres; ${ }^{5}$ En México, Miguel Bustamante hizo ver sus vertientes organizacionales. ${ }^{6}$ El equipo de la Organización Mundial de la Salud liderado por Douglas Bettcher, ${ }^{3}$ profundizó en las funciones esenciales de la salud pública, dándole a la regulación el espacio específico que le corresponde dentro del sistema de salud.

La regulación en salud se encuentra en un contexto amplio del sistema público y económico, cuyo desarrollo teórico y empírico ha sido profundizado por economistas acreedores de premios Nobel. Amartya Sen ${ }^{7}$ subrayó la importancia del Estado en la protección a la sociedad y la equidad. Douglass North ${ }^{8}$ abordó la economía de las instituciones, en este caso las regulatorias, y su importancia para el desarrollo; Jean Tirole, ${ }^{9}$ la necesidad de la regulación frente a las fallas del mercado; y George Stigler, ${ }^{10}$ la captura regulatoria por intereses especiales.

En un documento técnico de referencia se describe cómo la acción de protección se organiza en un proceso regulatorio (Figura 1). ${ }^{11}$ Parte del análisis de riesgo se continúa con la determinación de su manejo, incluyendo el establecimiento de reglas y estándares, cuyo proceso, cumplimiento e impacto deben ser vigilados y sometidos a evaluación, en especial la efectividad en distintos sectores poblacionales. ${ }^{12}$

El proceso regulatorio está basado en la ciencia e incluye el escrutinio por pares, sine equa non lo es para el análisis de riesgo. ${ }^{13}$ Para ello se retoma el conocimiento de la identificación del peligro y las rutas de exposición, por ejemplo, fármacos, sustancias tóxicas y elementos agregados a los alimentos, entre otros. Este saber es global y a él contribuyen los metaanálisis y guías formuladas por organismos multilaterales internacionales como la Agencia de Investigación del Cáncer, la Organización Mundial de la Salud, CODEX, National Institute for Health and Care Excellence, Network Time Protocol, Environmental Protection Agency y Food and Drugs Administration.

Pero este conocimiento debe ser analizado conforme a las condiciones locales, particularidades sociales, ambientales, nutricionales, culturales y epidemiológicas, para caracterizar los riesgos específicos según el contexto, ${ }^{13}$ por ejemplo, los problemas de obesidad infantil, contingencias atmosféricas o malformaciones congénitas requieren acciones específicas según su prevalencia en los distintos países.

Con base en modelos predictivos se definen instrumentos de manejo de riesgo, incluyendo las acciones 




Figura 1. El proceso regulatorio en salud.

de promoción de la salud, de prevención clínica y elaboración de reglas y normas regulatorias. La selección de los instrumentos debe fundamentarse en estudios de costo-efectividad. ${ }^{14}$ Una vez decidida la ruta regulatoria, el desarrollo de una norma, su implementación y cumplimiento se convierten en el componente crítico de la efectividad: es ahí cuando sucede la acción regulatoria. El monitoreo y la evaluación del proceso deben ser integrales y proporcionar evidencia de costo-efectividad o elementos para llevar a cabo ajustes. Finalmente, es la evaluación del impacto económico y epidemiológico y la evaluación retrospectiva de la efectividad regulatoria, en especial la evaluación expandida ${ }^{12}$ a identificar el impacto en los diferentes grupos sociales, lo que da el cuerpo de evidencia al proceso regulatorio.
Es principio fundamental de la arquitectura, enunciado por Louis Sullivan a finales del siglo XIX, que la forma sigue a la función. ${ }^{15}$ Como sucede en otros ámbitos del sistema de salud, frecuentemente se omite la consideración de las estructuras para que las acciones se realicen con calidad y efectividad requeridas, pero, a diferencia de la atención médica, la ausencia de diseño y organización de calidad y efectividad regulatorias afecta a millones de personas en el corto plazo, de ahí su importancia.

El proceso para el ejercicio de la función regulatoria en salud requiere una arquitectura tecnológica y humana, que incluya tanto profesionales altamente formados como equipos de laboratorio y de campo, para tener una institución competente, así como una arquitectura financiera que haga viable, autónoma, 


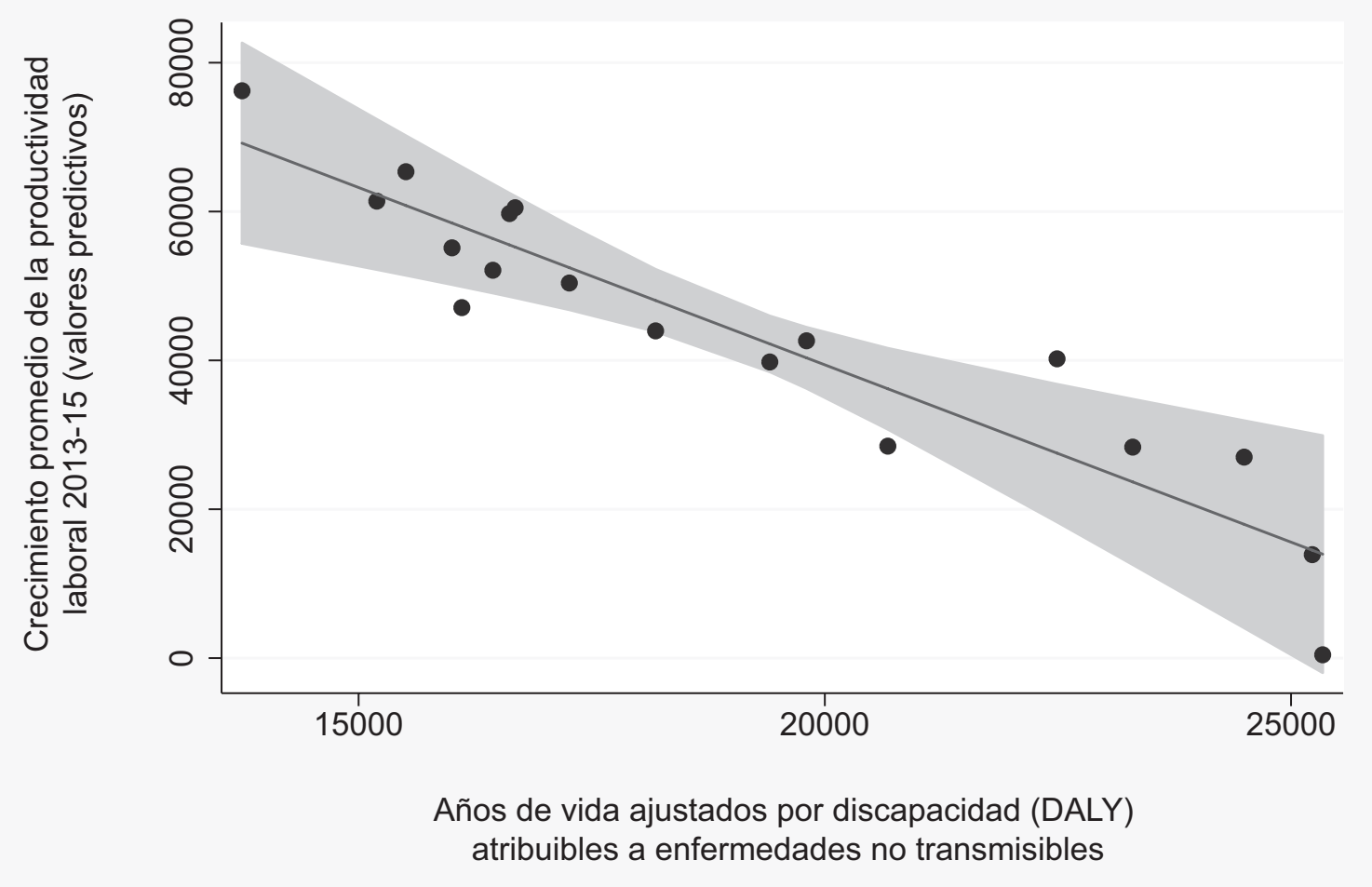

Figura 2. Influencia de los años de vida de salud perdidos por enfermedades no transmisibles sobre el crecimiento de la productividad laboral en la región de las Américas, 2013-2015.

equitativa su función y elimine los subsidios indirectos que distorsionen el mercado. Para ello es indispensable conocer los costos de las operaciones en cada paso del proceso y examinar las distintas fuentes de flujo financiero, provenientes tanto de los derechos por las partes reguladas, por sanciones, como por los recursos asignados presupuestalmente vía impuestos generales. Requiere un andamiaje organizacional para su eficiencia, equidad y no apropiación administrativa de sectores regulados o componentes del proceso, uno legal que provea certidumbre a reguladores, regulados y a sociedad, y uno de gobernanza, para mayor integridad de la intervención estatal.

La gobernanza se refiere a las formas de inclusión de los actores interesados o afectados, buscando la eficacia, calidad y buena orientación del Estado en cada etapa del proceso regulatorio..$^{16}$ Incluye mecanismos, prácticas y estructuras institucionales a través de las cuales se ejerce la autoridad, se manejan los conflictos de interés y se logra que los actores gubernamentales y no gubernamentales contribuyan con conocimiento y práctica, participen sin captura regulatoria, rindiendo cuentas. Tiene que ver con la relación del sector salud como usuario y prestador de productos y servicios; con la relación con otros sectores y órdenes del ejecutivo y del legislativo, con el involucramiento equitativo de la sociedad civil interesada, los sectores regulados y la academia.

En el decreto de creación de la Comisión Federal para la Protección contra Riesgos Sanitarios (Cofepris) del 5 de julio de $2001^{17}$ se explicita una Comisión Mixta; en las fracciones I y II se menciona que la conformarán agrupaciones, asociaciones, cámaras o empresas del sector privado, social, productivo y de servicios relacionados directamente con las actividades objeto de la Comisión, así como por personas que se distingan por sus méritos académicos o científicos en áreas relacionadas con el ámbito de competencia de la Comisión. Su función fundamental será conocer y opinar sobre las políticas regulatorias y de fomento que determine o implante la Comisión, así como sobre las prioridades en la normalización, 


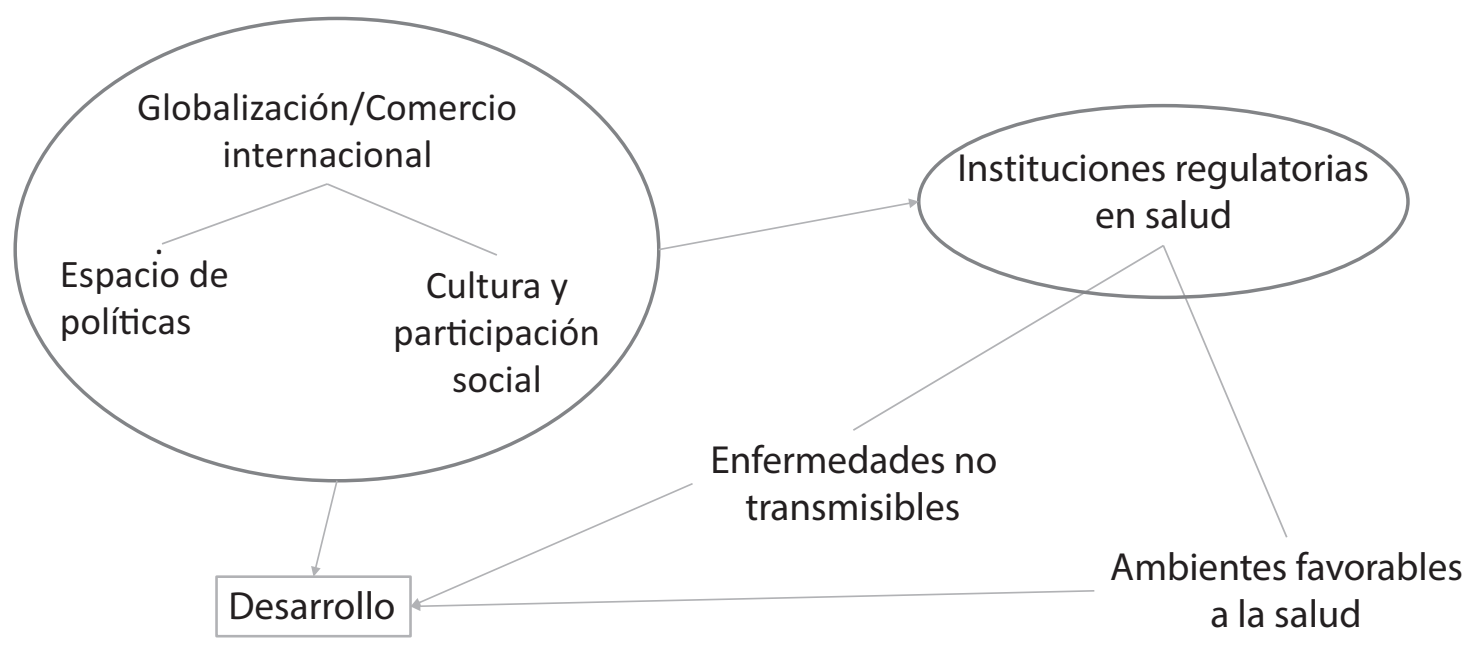

Figura 3. Relación de las enfermedades no transmisibles, el desarrollo y las instituciones regulatorias en salud.

debido a los riesgos a la salud pública implicados. Su reglamento ${ }^{18}$ incluyó las Comisiones Científica y Consultiva Mixta, lo cual constituyó un primer esbozo de ese andamiaje de gobernanza, cuya continuidad no ha sido llevada a cabo.

\section{Regulación en salud, competitividad y desarrollo}

Al ser un bien común, el impacto de la regulación en la economía y competitividad se da por el beneficio universal de la protección a la salud que brinda, pero también por la diversidad y magnitud económica de los sectores regulados, cuyos productos y servicios puede impulsar.

En un análisis transversal de 31 países de la región de las Américas (Santos-Burgoa C. Health regulatory institutional capacity effect on NCDs and development in the context of globalization and trade in the region of the Americas, sometido para publicación, 2017), para el periodo 2013 a 2015 se identificó que a menor carga de enfermedad (Figura 2), mayor crecimiento en la productividad laboral. Los análisis en este estudio hacen concluir que a mayor capacidad regulatoria en salud, menor la carga de enfermedad y discapacidad por enfermedades no transmisibles, las cuales conllevan el mayor peso de salud en la región (Figura 3). A su vez, las instituciones regulatorias reducen las enfermedades no transmisibles, que sin control quebrantan el desarrollo e incrementan el gasto en salud.

Los datos empíricos muestran cómo la intensificación de la globalización y liberalización comercial favorecen el espacio de políticas, la cultura y la participación social, como ha sucedido en los más de 20 años del Tratado de Libre Comercio de América del Norte; estos a su vez influyen en la creciente capacidad regulatoria en salud.

Al analizar la dimensión económica ${ }^{19}$ de los sectores regulados por salud en la región, estos comprenden al menos $17 \%$ del comercio internacional, lo que implica que en las negociones de comercio internacional es fundamental contar con autoridades sanitarias que contribuyan a los acuerdos y a la coherencia regulatoria, ${ }^{20}$ reduciendo barreras $s$ innecesarias $y$ estructurando la colaboración que asegure la protección poblacional y la competitividad. 


\title{
La regulación sanitaria en México: Comisión Federal para la Protección contra Riesgos Sanitarios
}

\author{
Julio Salvador Sánchez-Tépoz, Juan Carlos Gallaga-Solórzano, Rocío del Carmen Alatorre Eden Wynter \\ y Armida Zúñiga-Estrada
}

\section{Introducción}

El 5 de julio del 2001 se emitió el decreto de creación de la Comisión Federal para la Protección contra Riesgos Sanitarios (Cofepris) como autoridad sanitaria en México, ${ }^{17}$ con la finalidad de fortalecer la protección contra los riesgos sanitarios, con una orientación en política de salud pública acorde con el contexto internacional, la transición epidemiológica de la población mexicana y el desarrollo del país, permitiendo a la autoridad el efectivo ejercicio de sus atribuciones en un ámbito de transparencia, certidumbre, participación social y con sustento en la mejor evidencia técnica y científica disponible.

Esta unidad se creó con el objetivo de integrar la totalidad de las funciones de control sanitario en un solo órgano que, como parte de la Secretaría de Salud, homologue las políticas y que disponga de autonomía técnica, administrativa y operativa que le permita tomar decisiones con mayor rapidez, eficiencia y flexibilidad. ${ }^{18}$

Este nuevo órgano se constituyó por las Direcciones Generales de Medicamentos y Tecnologías para la Salud, Control Sanitario de Productos y Servicios, Salud Ambiental, Laboratorio Nacional de Salud Pública y Control Sanitario de la Publicidad, que se encontraban adscritas a la extinta Subsecretaría de Regulación y Fomento Sanitario.

El 30 de junio de 2003, en México se publicaron diversas modificaciones a la Ley General de Salud, ${ }^{21}$ mediante las cuales se fortaleció el carácter rector de la Cofepris que, de acuerdo con el artículo 3 de su reglamento interno,$^{18}$ tiene atribuciones para regular:

a) Establecimientos de salud, disposición de órganos, tejidos, células de seres humanos y sus componentes y disposición de sangre.

b) Medicamentos, remedios herbolarios y otros insumos para la salud.

c) Alimentos y suplementos alimenticios.

d) Bebidas alcohólicas y bebidas no alcohólicas.

e) Productos de perfumería, belleza y aseo.

f) Tabaco.

g) Plaguicidas y fertilizantes.

h) Nutrientes vegetales.

i) Sustancias tóxicas o peligrosas para la salud. j) Químicos esenciales, precursores químicos, estupefacientes y psicotrópicos.

k) Productos biotecnológicos.

l) Materias primas y aditivos que intervengan en la elaboración de los productos señalados en los incisos b) a k), así como los establecimientos dedicados al proceso o almacenamiento de estos.

m)Fuentes de radiación ionizante para uso médico.

n) Efectos nocivos de los factores ambientales en la salud humana.

o) Salud ocupacional.

p) Saneamiento básico.

q) Importaciones y exportaciones de los productos a que se refiere la fracción II del artículo 17 bis de la Ley.

r) Publicidad y promoción de las actividades, productos y servicios a que se refiere la Ley y demás disposiciones aplicables.

s) Sanidad internacional, salvo en las materias exceptuadas por la Ley.

t) En general, los requisitos de condición sanitaria que deben cubrir los procesos, productos, métodos, instalaciones, servicios o actividades relacionados con las materias descritas, en los términos de la Ley y demás disposiciones aplicables.

\section{Sectores e industrias regulados}

La Comisión es una agencia con amplias competencias regulatorias, cuya actividad primordial es la prevención contra riesgos sanitarios y se relaciona con los sectores industriales, que representan $9.8 \%$ del producto interno bruto y $10.94 \%$ del comercio exterior.

La Cofepris regula 44 centavos de cada peso gastado por los hogares en México, a través de tres categorías: alimentos, bebidas y tabaco; artículos para el cuidado de la salud y artículos para cuidado personal.

\section{Regulación sanitaria}

La Cofepris está encargada de regular diversos procesos:

- Registros de medicamentos, dispositivos médicos, plaguicidas y nutrientes vegetales. 
Tabla 1. Vía de autorización de registros liberados

\begin{tabular}{lcccc}
\hline Evento & Nuevos medicamentos & Acuerdo de equivalencias & México-primer país de comercialización & Total \\
\hline Primero & 31 & 0 & 0 & 31 \\
Segundo & 29 & 0 & 0 & 29 \\
Tercero & 22 & 5 & 1 & 28 \\
Cuarto & 11 & 1 & 2 & 13 \\
Quinto & 26 & 4 & 0 & 32 \\
Sexto & 13 & 4 & 1 & 17 \\
Séptimo & 15 & 11 & 1 & 27 \\
Octavo & 31 & 4 & 1 & 36 \\
Noveno & 32 & 30 & 7 & 276 \\
Total & 210 & 59 & & 63 \\
\hline
\end{tabular}

- Licencias para hospitales y clínicas de salud.

- Comercio de productos cosméticos y de belleza.

- Avisos de funcionamiento de farmacias y consultorios.

- Avisos de publicidad en medicamentos y alimentos.

- Vigilancia sanitaria, aseguramiento, multas y sanciones sobre productos como alcohol, tabaco y medicamentos.

- Aprobación de protocolos para la investigación clínica.

- Muestreo y vigilancia de vacunas.

- Vigilancia en cumplimiento de etiquetado en alimentos.

- Permisos de importación y exportación de insumos para la salud.

\section{Regulación de medicamentos e insumos para la salud}

Los medicamentos y demás insumos deben atender los patrones de morbilidad y mortalidad en el país. En el Plan Nacional de Desarrollo 2013-2018 se proyectó hacer de México una sociedad con goce de los mandatos otorgados por la Constitución, para lo cual se estableció como objetivo principal fortalecer el acceso efectivo y oportuno de la población a un mercado farmacéutico debidamente abastecido con productos seguros, eficaces y de calidad.

La política farmacéutica implementada por la Cofepris se encuentra alineada a cuatro ejes fundamentales: un ente regulatorio que garantice la seguridad, calidad y eficacia de los medicamentos; un esquema solvente de autorización de registros sanitarios; la eliminación de las barreras de entrada al mercado a productos seguros, de calidad y eficaces y la homologación del regulador con las mejores prácticas internacionales.

En los últimos años, en México se ha liberado 37 sustancias activas que corresponden a 491 nuevos registros de medicamentos genéricos que atienden $71 \%$ de las causas de mortalidad en la población mexicana. De esta forma, la política de genéricos ha permitido una reducción promedio de $55 \%$ en los precios de los medicamentos, un promedio un ahorro de $\$ 1446$ pesos para el consumidor, un ahorro acumulado de 24632 millones de pesos y 1998202 pacientes adicionales atendidos. No se dispone del registro internacional de otra estrategia de liberación de genéricos a tal escala ni en tan reducido periodo. También se han liberado 276 nuevas moléculas que abarcan 23 grupos terapéuticos. México es el primer país de comercialización de siete moléculas (Tabla 1). ${ }^{22}$

\section{Dispositivos médicos}

De acuerdo con el artículo 82 del Reglamento para Insumos de la Salud, ${ }^{23}$ para la producción, venta y distribución de los dispositivos médicos u otros insumos se requiere registro sanitario.

Durante 2016, la Cofepris atendió alrededor de 18000 trámites de registros para dispositivos médicos. La solicitud de registros sanitarios para la comercialización e introducción de dispositivos médicos ha aumentado como consecuencia del avance tecnológico y la globalización. Por ello, la Cofepris ha iniciado programas con el objetivo de generar mecanismos regulatorios más eficientes y homologarlos con criterios internacionales. 


\section{Terceros autorizados}

Los terceros autorizados son figuras que actúan como auxiliares de la regulación y tienen como objetivo apoyar la autoridad sanitaria en el desahogo de trámites, permitiendo la disminución de cargas administrativas y una mejor atención a los usuarios. Entre los beneficios del esquema se pueden mencionar la ampliación de cobertura en atención a solicitudes de registro, el incremento del número de dictaminadores externos y la disposición de 19 unidades de verificación externas.

\section{Indicadores de las industrias reguladas 2008-2014}

Entre 2008 y 2014, la industria alimentaria alcanzó 673 mil millones de pesos; entre 2008 y 2010 , la tasa de crecimiento fue de $14.7 \%$; entre 2011 y 2014, de $18.3 \%$. Entre 2008 y 2014, la industria de bebidas y tabaco alcanzó un valor de 149 mil millones de pesos; entre 2008 y 2010 la tasa de crecimiento fue de $11.4 \%$; entre 2011 y 2014 , de $20.4 \%$. En cuanto a la industria química, entre 2008 y 2014 alcanzó un valor de 338 mil millones de pesos; entre 2008 y 2010 la tasa de crecimiento fue de $2.1 \%$ y entre 2011 y 2014 , de $23.5 \%$. El mercado farmacéutico alcanzó entre 2008 y 2014 un valor de 195 mil millones de pesos; entre 2008 y 2010 la tasa de crecimiento fue de $1.8 \%$ y entre 2011 y 2014 , de $13.2 \%$. $^{24}$

\section{Logros de la autoridad sanitaria}

Entre los logros alcanzados por la Cofepris a lo largo de 17 años, se encuentran los siguientes:

- Reconocimiento por la Organización Mundial de la Salud en materia de vacunas y por parte de la Organización Panamericana de la Salud en materia de medicamentos y vacunas, así como múltiples acuerdos internacionales con otras agencias.

- Implementación de procesos regulatorios con base en el riesgo, lo que ha permitido mejorar la simplificación de estos.

- Implementación de un sistema federal sanitario con rectoría a nivel estatal.

- Participación activa de la sociedad civil (agentes regulados, organizaciones no gubernamentales, la academia) en el diseño de las políticas públicas.

- Implementación de políticas regulatorias y no regulatorias mediante el fomento sanitario.
- Armonización para la puesta en práctica de los mejores estándares internacionales en regulación (benchmarking).

- Intercambio de información con agencias hermanas, tales como la European Medicine Agency, Food and Drug Administration, Health Canada, Foro de Cooperación Económica Asia-Pacífico.

\section{Conclusiones}

En los últimos años, la Cofepris ha centrado sus esfuerzos en la protección de riesgos sanitarios, enfocándose en el fortalecimiento del marco regulatorio, crecimiento de las materias con rezago medio y bajo, así como el manejo del presupuesto adecuado y manejable para una implementación efectiva de las actividades con base en el riesgo. Conforme el impacto, los logros, la factibilidad y las necesidades en materia de salud pública, la Cofepris considera las siguientes oportunidades para seguir contribuyendo a la protección de la salud de la población:

- A corto plazo, continuar con el fortalecimiento en materia de medicamentos, remedios herbolarios y otros insumos para la salud, tabaco, químicos Esenciales, precursores químicos, estupefacientes y psicotrópicos; productos biotecnológicos, importaciones y exportaciones de los productos a que se refiere la fracción II del artículo 17 bis de la Ley, la publicidad y promoción de las actividades, productos y servicios a que se refiere la Ley y demás disposiciones aplicables.

- A mediano plazo, fortalecer las estrategias relacionadas con la regulación de alimentos y suplementos alimenticios, bebidas alcohólicas y bebidas no alcohólicas, productos de perfumería, belleza y aseo; plaguicidas, fertilizantes y nutrientes vegetales.

- A largo plazo, el fortalecimiento del marco regulatorio sobre sustancias tóxicas o peligrosas para la salud, fuentes de radiación ionizante para uso médico, efectos nocivos de los factores ambientales en la salud humana, salud ocupacional y saneamiento básico.

\section{Agradecimientos}

Al personal de la Cofepris, por su alta capacidad técnica, compromiso y profesionalismo para cumplir a cabalidad las responsabilidades adquiridas como servidores públicos, así como a la doctora Hilda Durán Álvarez, por el apoyo editorial. 


\title{
Regulación en el tema de seguridad vial y los retos intersectoriales
}

\author{
Martha Hijar y Ricardo García-Sarubbi
}

\section{Introducción}

Definir lo "inaceptable" es uno de los retos de la salud pública. La muerte por accidentes viales siempre será una tragedia, pero se ha vuelto inaceptable a partir de la existencia y accesibilidad de las intervenciones para su prevención y control. Según el Secretariado Técnico de la CONAPRA, como consecuencia de la inseguridad vial para 2015 en México hubo 37228 muertes por accidentes (4.2/hora), de las cuales 16039 (43\%, 370/día, 15/hora) se debieron a incidentes de tránsito, que constituyeron $30 \%$ del peso de la enfermedad, además de que los accidentes en motociclistas han ido en incremento.

En el tema de mortalidad ${ }^{25}$ destaca el peso de la enfermedad que representan las lesiones, sobre todo el componente de muertes prematuras, ${ }^{26}$ es decir, el impacto en la población joven, y el incremento de los daños a la salud en el motociclista.

\section{Enfoque interdisciplinario y multisectorial}

La seguridad vial tendría que abordarse desde una perspectiva integral que incluya los aspectos urbanos, el transporte y los efectos en salud (Tabla 2). ${ }^{27}$

En este sentido, es importante resaltar que los efectos de dichas variables se traducen en cambios en el uso del suelo y la dependencia del automóvil, ambos asociados con daños a la salud, como obesidad, enfermedades crónicas (diabetes, cáncer), problemas cardiovasculares y salud mental. Todos estos efectos que, junto con los accidentes de tránsito, normalmente son analizados en forma aislada del entorno y de la interacción social. Una primera reflexión sería pensar en la necesidad de vincular las políticas públicas de salud, con las de transporte y vivienda, por mencionar algunas.

Ante este panorama, los instrumentos de regulación y política en seguridad vial representan un gran reto, ya que están asociados con la forma como la población puede y decide movilizarse y con la identificación de los sectores involucrados, como el de la educación y el laboral. En la Figura 4 se muestra cada sector responsable de las acciones en las que está dividido el gobierno; queda clara la gran dispersión de atribuciones. Todas las acciones de gobierno al final están permeadas por los determinantes sociales, así como por la disponibilidad y el acceso a los servicios de salud. Sin duda, el papel del sector de la salud en el tema de la seguridad vial ha sido decisivo, un ejemplo es el Informe Mundial sobre Seguridad Vial, publicado en $2004,{ }^{28}$ que estableció cuatro categorías de intervención: las dos últimas se identifican claramente en el ámbito del sector de la salud, la segunda es menos precisa, mientras que la primera, si bien se planteó como la de mayor potencial para reducir las muertes y lesiones, ha sido la de más baja implementación y en la que quedan claros los retos del trabajo intersectorial, incluyendo el sector privado dedicado a la producción y venta de automóviles, que desarrolla impresionantes campañas de venta. Lo anterior es particularmente preocupante debido al impacto negativo que la dependencia del automóvil tiene en muchas dimensiones de la salud. ${ }^{28}$

\section{Retos para regular desde salud el tema de seguridad vial}

En marzo de 2010, las Naciones Unidas proclamó el periodo 2011-2020 como el Decenio de Acción para la Seguridad Vial, ${ }^{29}$ con un único objetivo: estabilizar y luego reducir el número de muertes debidas al tránsito en todo el mundo, mediante el aumento de las actividades de prevención a nivel nacional, regional y mundial. Se da la batuta a la Organización Mundial de la Salud para la aplicación de esta resolución, con la cual México está comprometido a través de una Estrategia Nacional de Seguridad Vial, ${ }^{30}$ firmada por la Secretaría de salud y la Secretaria de Comunicaciones y Transportes.

Como se puede observar en la Figura 5, cada pilar que plantea la resolución requiere el trabajo intersectorial para implementar acciones, incluso en lo que se refiere a la atención médica pre y hospitalaria.

En el mundo, la seguridad vial como problema de salud pública se ha asumido como responsabilidad del sector del transporte, mientras que en México recayó en el Sector Salud a través de la creación, hace 31 


\begin{tabular}{ll}
\hline & $\begin{array}{l}\text { Disminución de la actividad física. Enfermedades } \\
\text { cardiovasculares, obesidad. }\end{array}$ \\
Cambios en el uso de suelo & Contaminación ambiental y asma. \\
Dependencia del automóvil & Impacto en el cambio climático \\
Efectos en los procesos sociales & Incremento de accidentes de tránsito. \\
& Incremento de peatones atropellados. \\
& Impacto en la salud mental. \\
\hline Fuente: Referencia 27. & Disminución, ausencia o descenso en el capital social. \\
\hline
\end{tabular}

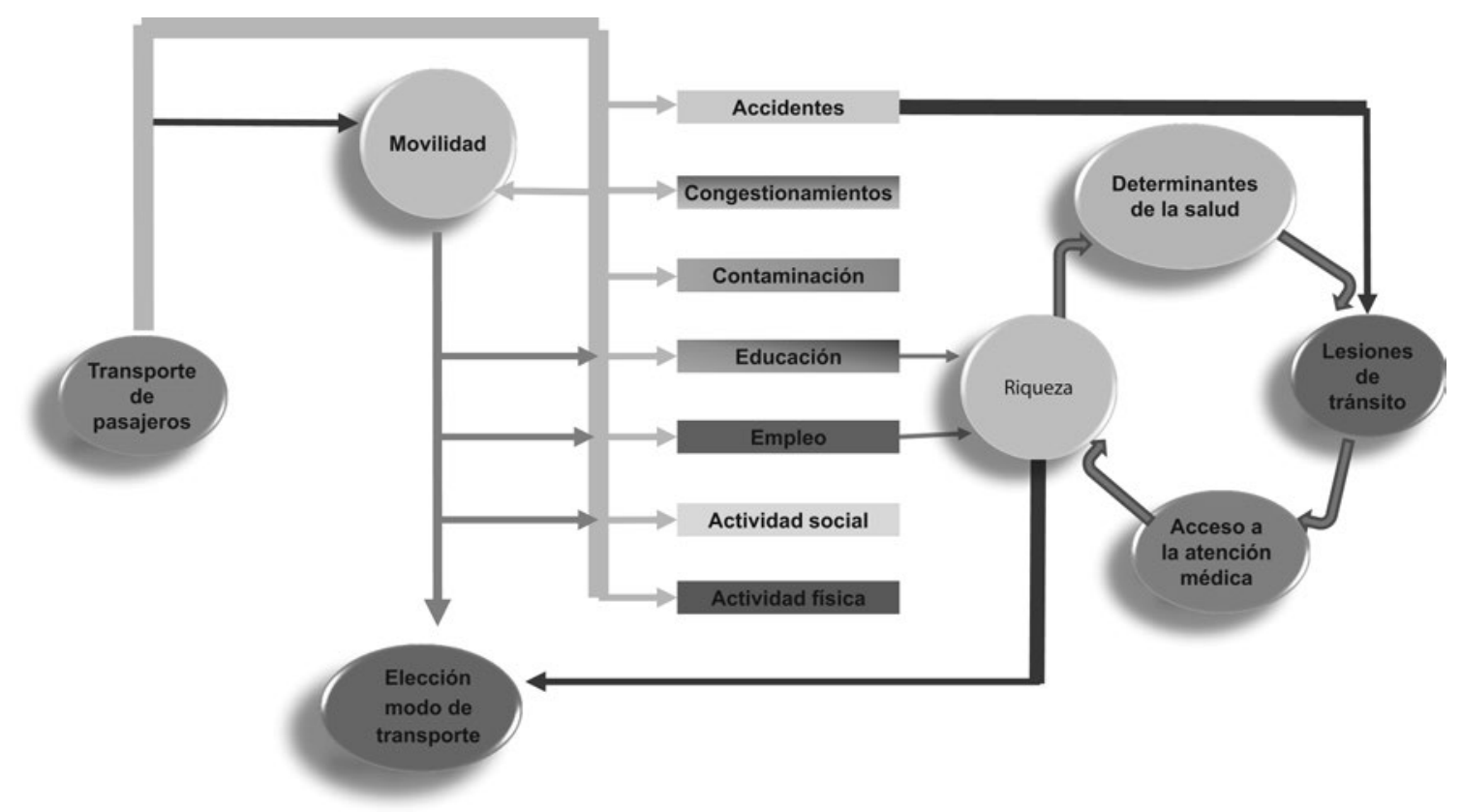

Figura 4. Sectores e instrumentos de regulación y política relacionados con la seguridad vial.

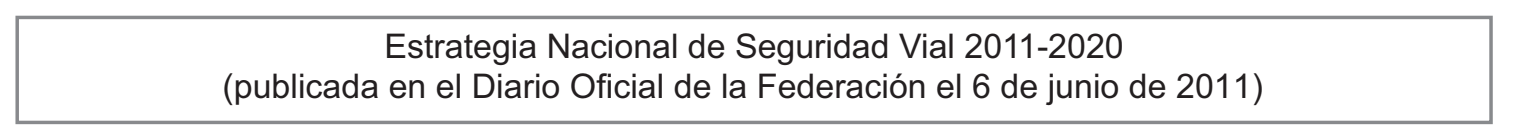

Cinco pilares para la acción
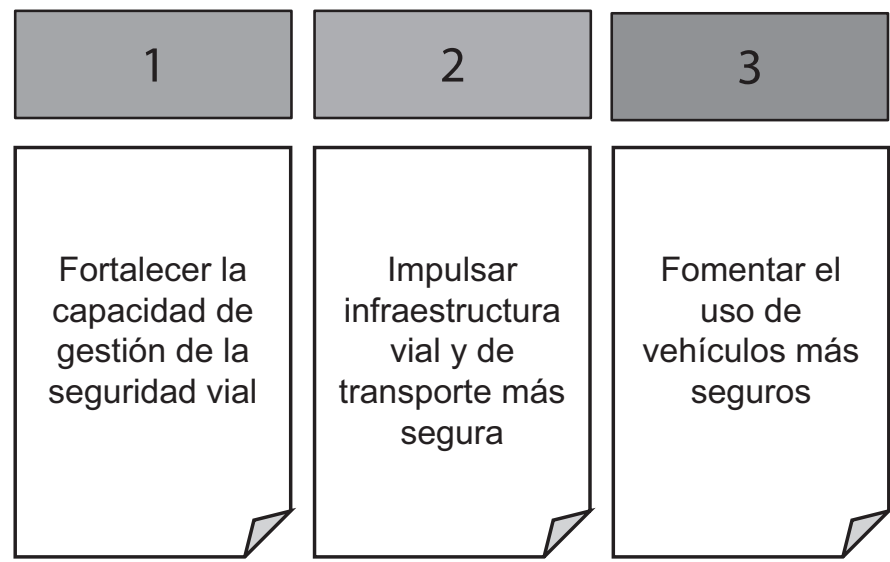
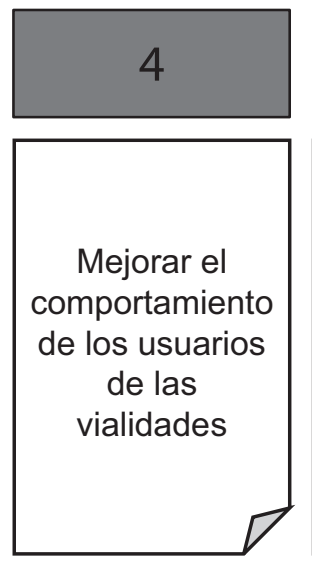

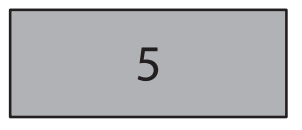

Impulsar la mejora de los servicios de atención médica prehospitalaria y hospitalaria

Figura 5. Pilares de acción del Decenio de Acción por la Seguridad Vial propuestos por la Organización Mundial de la Salud. 
años, del CONAPRA, ${ }^{31}$ órgano colegiado con representación multisectorial presidido por el secretario de Salud, el cual se instituyó antes de que la Organización Mundial de la Salud tuviera un área específica en este tema.

El Conapra cuenta con un Secretariado Técnico (STConapra), que depende de la Subsecretaría de Prevención y Promoción de la Salud. A través de este Secretariado, en los estados se ha promovido la instalación de consejos estatales a lo largo del país, responsables de aplicar el Programa de Seguridad Vial ${ }^{32}$ que forma parte del Programa Sectorial de Salud, con financiamiento federal desde el 2008 y en algunos casos estatal.

¿Por qué en México la prevención de accidentes, específicamente el Programa de Seguridad Vial, se encuentran ubicado en el Sector Salud? Algunas razones pueden ser la falta de acción de otros sectores involucrados, porque en él se generan indicadores de impacto de las intervenciones y porque la inseguridad vial impacta en todas las esferas del sistema de salud.

Sin duda, el impacto de los daños generados por la inseguridad vial en el sistema de salud fue una de las principales razones que impulsaron la creación del consejo en 1986, periodo durante el cual el doctor Guillermo Soberón Acevedo era el Secretario de Salud en México.

\section{Acciones de la rectoría del Sector Salud en México}

Entre las acciones del Programa de Seguridad Vial relacionadas con la regulación se encuentran promover leyes, reglamentos, normas y reformas legales para mejorar la seguridad vial en el país, con énfasis en los estados y municipios. Con un problema tan serio como la inseguridad vial y sus consecuencias, sería necesario promulgar una Ley General de Seguridad Vial que, desde el punto de vista de salud pública, contribuya a salvaguardar el derecho a este bien, jurídicamente tutelado por la Constitución General, para quienes se encuentran en el territorio nacional, incluido el carácter que a este concepto de salud pública le dan los principios rectores de los derechos humanos.

Como ejemplo de lo anterior y en respuesta al tema de los accidentes en motocicleta, el Secretariado Técnico de la CONAPRA presentó ante las instancias correspondientes el PROY-NOM-206-SCFI/SSA22016, Proyecto de norma oficial mexicana de cascos de seguridad para la prevención y atención inmediata de lesiones en la cabeza de motociclistas, acciones de promoción de la salud, especificaciones de seguridad y métodos de prueba, información comercial y etiquetado, ${ }^{33}$ promovido desde la Secretaría de Salud para contar con cascos homologados para motociclistas, que tuvo que salir en conjunto con la Secretaría de Economía y que está próximo a publicarse en el Diario Oficial de la Federación.

Hay que recordar que es el municipio a quien la Constitución de los Estados Unidos Mexicanos ${ }^{34}$ confiere la autoridad en el tema del tránsito, por lo que desde 2016 se trabaja con la red de municipios por la salud, incorporando el tema de seguridad vial, con lo que se ha logrado disponer, además de los consejos estatales, con 23 consejos municipales que cuentan con un plan de seguridad vial, en el cual el tema de regulación tiene un peso importante.

\section{Rezagos en la regulación de la seguridad vial}

A pesar del contar con un consejo con participación intersectorial y de los incipientes avances en la reducción de la mortalidad, los rezagos en la regulación de la seguridad vial podrían resumirse en los siguientes:

- Los accidentes viales no constituyen una presión política para los tomadores de decisiones de salud y la mayor parte de las veces su resolución se reduce a la dotación de ambulancias y camas hospitalarias.

- Los accidentes viales no se perciben como un problema que deba prevenirse desde el Sector Salud.

- La cantidad de recursos que es necesario derivar a los problemas agudos de salud. El Programa de Seguridad Vial, al depender de instancias en las cuales se atienden problemas de alto impacto político, como las enfermedades transmisibles o las infecciosas, no resulta prioritario a pesar de su importancia en los indicadores de salud y queda relegado desde su atención hasta la designación de recursos financieros.

- Requiere la voluntad política de las autoridades de todos los niveles de gobierno. El derecho a la salud consagrado en la Constitución Política de los Estados Unidos Mexicanos impone deberes y obligaciones a todos los poderes públicos del Estado, que van desde el Legislativo hasta el Jurídico. 
- Los incidentes viales continúan siendo percibidos como aleatorios, ${ }^{35}$ incluso por los trabajadores de la salud y resulta difícil convencer a la población de que las lesiones no intencionales "no son accidentes". Lo anterior se basa en que todas las causas de lesión pueden ser analizadas a partir de la relación causa-efecto, base de la ciencia, y que al estudiar y conocer las causas es posible identificar intervenciones de prevención. La sencillez de las medidas preventivas hace que sean percibidas como innecesarias $y$, por lo tanto, como "inaceptables", de ahí uno de los grandes retos de la salud pública. ${ }^{36}$

\section{La necesaria interacción intra e intersectorial}

En la medida en que las políticas dirigidas a buscar soluciones al creciente problema de las lesiones debidas al tránsito se encuentren dentro de la responsabilidad de un solo sector, será limitado el impacto que dichas políticas generen. En México, el objetivo general de la política en este tema ha sido que la reducción de las muertes de accidente en la vía pública se debe realizar a partir de los cinco pilares de intervenciones prescritas en la política internacional: la gestión de la seguridad vial, vehículos más seguros, usuarios de la carretera más seguros y mejorar la atención médica posterior al accidente. El primer problema con este enfoque es que se ignora que los eventos que producen lesiones en la vía pública son producto de factores sociales, económicos y ambientales mencionados arriba y que no pueden ser cambiados en el aislamiento de estos contextos. Focalizarse solo en reducir los eventos que producen lesiones en la vía pública podría mejorar los resultados de los accidentes, sin embargo, las condiciones sistémicas que contribuyen a la ocurrencia de dichos eventos permanecen intactas. El segundo problema es que las lesiones debidas al tránsito son solo uno de los resultados de salud derivados del transporte. Centrarse únicamente en la reducción de lesiones es de poco beneficio de la salud pública, sobre todo si se alcanza a expensas del deterioro en otros aspectos de la salud. ${ }^{36}$

Expongo los retos que desde la Academia Nacional de Medicina habría que enfrentar:

- Promover la discusión con enfoque interdisciplinario y multisectorial

- Comenzar a dialogar con otros sectores del sector salud como los ambientales o los de estructura urbana e incluso con otras Academias. 


\title{
Avanzando las fronteras regulatorias para enfermedades crónicas
}

\author{
Juan Ángel Rivera-Dommarco, Claudia Gabriela García-Chávez y Ana Carolina Ariza
}

\section{Introducción}

Las enfermedades crónicas no transmisibles (ECNT) en la población constituyen uno de los mayores retos para el sistema de salud por su alta prevalencia, contribución a la mortalidad y elevado costo de su tratamiento. ${ }^{26}$

Las ECNT provocan alrededor de 40 millones de muertes cada año, es decir, $70 \%$ en el ámbito mundial; más de 15 millones de esas muertes ocurren de forma prematura, en etapas de la vida en que no deberían ocurrir. Además, más de $80 \%$ acontecen en países en desarrollo, ${ }^{37}$ de tal forma que para países como México es fundamental su prevención. ${ }^{38,39}$

Existen diversos factores de riesgo genéticos, fisiológicos, ambientales o conductuales que pueden contribuir al desarrollo de las ECNT, particularmente destacan cuatro de naturaleza modificable: consumo de tabaco, dietas no saludables, uso nocivo de alcohol e inactividad física. ${ }^{40}$ Aunque estos factores son individuales, se debe reconocer la responsabilidad colectiva en la prevención de las ECNT ${ }^{41}$ y, por lo tanto, la necesidad de la intervención del Estado.

\section{La regulación en la prevención y control de las ECNT}

Un individuo puede o no optar por modificar alguno de los cuatro factores de riesgo (Figura 6); para ello es importante conocer los efectos positivos de una conducta saludable y conocer para adoptarla. Sin embargo, la educación y el conocimiento no son suficientes debido a los ambientes promotores de ECNT, que interfieren con la adopción de comportamientos saludables.

Como parte de los ambientes promotores se encuentran los factores del entorno que determinan las conductas, donde influye la disponibilidad, la accesibilidad, los precios, la publicidad y la información sobre productos no saludables (como alcohol, tabaco, alimentos con alta densidad energética y pobre en nutrimentos (denominados popularmente como "comida chatarra"), bebidas azucaradas) y saludables (agua, oportunidades de actividad física).

Las escuelas son ejemplo de entornos en los que existen factores que condicionan la adopción de con- alimentos no nutritivos, tabaco, bebidas azucaradas a un precio similar al del agua embotellada y no disponen de bebederos de agua. ${ }^{42}$ En los hogares existe exposición a la publicidad de bebidas azucaradas en televisión u otras pantallas. ${ }^{43}$

En ambientes que propician conductas poco saludables es necesaria una regulación que convierta las conductas saludables en opciones viables y fáciles de adoptar. La conducta saludable se torna fácil, accesible y viable cuando se logran entornos libres de bebidas azucaradas, alimentos no nutritivos, alcohol o tabaco; con disponibilidad gratuita de agua y precios elevados de tabaco y bebidas azucaradas, aunados a la restricción de su publicidad en diversos medios de comunicación.

Una vez regulado el ambiente y logrando entornos saludables, con educación, motivación y conocimiento, el individuo podrá optar más fácilmente por la conducta saludable. La aspiración final es que las conductas saludables se conviertan en norma social y posiblemente entonces ya no sea necesaria la regulación.

La regulación es importante en el control y prevención de enfermedades; además de la educación, es indispensable la modificación del entorno físico y mediático. Como herramienta para la promoción de comportamientos saludables puede instaurarse a través de los diferentes niveles de gobierno: en el nivel ejecutivo mediante acuerdos, decretos e instancias, como la Cofepris; en el legislativo mediante leyes, por ejemplo, la ley de ingresos con el impuesto especial sobre producción y servicios (IEPS) a bebidas azucaradas.

También se requieren enfoques multisectoriales en la regulación, es decir, la participación de sectores de salud, comercio, agricultura, educación, desarrollo social, radio y televisión para incidir en actividades como la publicidad, el etiquetado, los subsidios agrícolas, los impuestos, la orientación en programas sociales. Se necesita influir en todos los entornos (escuela, ámbito público, edificios gubernamentales, mercados) para que conduzcan a la adopción de conductas saludables.

\section{Evidencia científica para el diseño de procesos regulatorios}

El uso de la evidencia científica es de suma importancia para orientar la regulación. En México, resalta el caso 


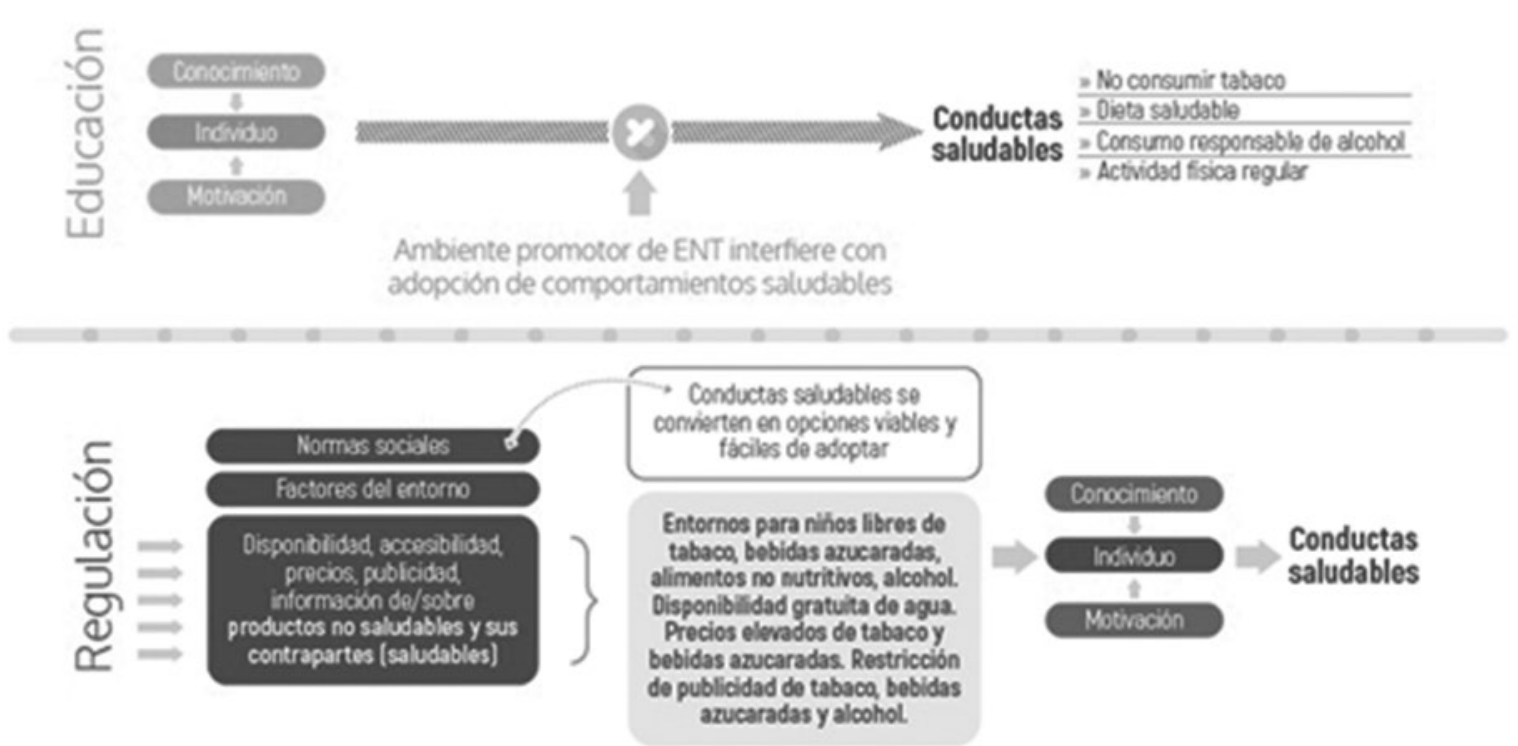

Figura 6. Reconocimiento de la responsabilidad colectiva en la prevención de enfermedades no transmisibles y de la necesidad de eliminar intervención del Estado. Modificado de referencia ${ }^{40}$.

de las bebidas azucaradas; su consumo se reguló mediante la ley de ingresos promulgada en septiembre de 2013 y se impulsó la aplicación de impuestos de bebidas azucaradas y de alimentos con una alta densidad energética. Para ello se partió de la evidencia científica de eliminar metaanálisis sobre el aumento en el riesgo de obesidad y diabetes debido al consumo de bebidas azucaradas. ${ }^{44}$ Más de $65 \%$ de la población mexicana rebasa el límite superior de consumo de azúcar adicionada recomendado por la Organización Mundial de la Salud;45 las bebidas azucaradas aportan casi $70 \%$ del azúcar adicionada que consumen los mexicanos. ${ }^{46,47}$ La demanda de bebidas azucaradas es elástica; los sustitutos de las bebidas azucaradas son el agua y las bebidas saludables, lo que se determina a través de la estimación de elasticidades cruzadas.48 Otra información importante para la adopción de medidas fiscales es la recaudación de impuestos en diferentes escenarios. Toda esta evidencia sirvió como base para la adopción del impuesto a las bebidas azucaradas, ejemplo del uso de la evidencia para el diseño de políticas regulatorias.

\section{Gobernanza del proceso regulatorio y los diferentes actores}

En términos de gobernanza del proceso regulatorio y el papel de sus diferentes actores, el poder ejecutivo participa con acuerdos por los que se establecen lineamientos en relación con el etiquetado de los productos, su expendio, distribución y regulación de la publicidad. El poder legislativo participa con la emisión de leyes específicas (IEPS) y generales (etiquetado) que pudieran afectar distintas medidas para disminuir riesgos. La academia debe generar evidencia científica que sustente la regulación para la salud mientras que la sociedad civil debe generar la demanda en materia de regulación para mejorar la salud e influir en el legislativo y el ejecutivo. Finalmente, la industria tiene la función de implementar las regulaciones y las leyes respecto a sus productos. La participación de los distintos sectores debe ser implementada y gobernada mediante procesos para lograr un manejo transparente, ya que en esta área existe un gran potencial de conflictos de interés.

\section{Monitoreo y evaluación independiente de las acciones regulatorias}

Es indispensable evaluar las medidas regulatorias en diferentes momentos, desde el diseño e implementación hasta los resultados y efectos (Tabla 3).

La evaluación de diseño es relativamente de bajo costo, simplemente se evalúa la política. Por ejemplo, en México, al revisar el diseño en publicidad puede identificarse que los horarios definidos para la aplicación de la regulación son inadecuados, ya que a ciertas horas del día o en ciertos programas de adultos existe audiencia infantil, que no debería estar expuesta a esos programas o su publicidad. ${ }^{49}$ Otro ejemplo es el etiquetado, cuya regulación establece un límite en el contenido de azúcar, que rebasa los límites establecidos por la Organización Mundial de la Salud..$^{50}$ Una ventaja de la evaluación de diseño es que no se necesita invertir mucho dinero para realizarla e identificar áreas de mejora. 
Gaceta Médica de México. 2018;154

Tabla 3. Importancia del monitoreo y la evaluación independientes de las acciones regulatorias

\begin{tabular}{|c|c|c|}
\hline Tipo de evaluación & Tipo de regulación & Ejemplos de resultados \\
\hline Evaluación de diseño & $\begin{array}{l}\text { Regulación de publicidad. } \\
\text { Etiquetado frontal. }\end{array}$ & $\begin{array}{l}\text { Gran audiencia de niños en ciertos horarios ( } 7-10 \text { p. m.) } \\
\text { y programas (telenovelas) no regulados. } \\
\text { Efecto poco probable en selección, compra y } \\
\text { reformulación. } \\
\text { Límite por arriba del recomendado por la Organización } \\
\text { Mundial de la Salud. }\end{array}$ \\
\hline Evaluación de implementación & $\begin{array}{l}\text { Regulación de publicidad. } \\
\text { Regulación de bebidas y alimentos en las } \\
\text { escuelas. }\end{array}$ & $\begin{array}{l}\text { En general, la regulación es acorde con las reglas. } \\
\text { Avances en la restricción de bebidas azucaradas } \\
\text { carbonatadas, pero menor en bebidas no carbonatadas. }\end{array}$ \\
\hline Evaluación de resultados & $\begin{array}{l}\text { Evaluación de compras de bebidas y } \\
\text { alimentos con y sin impuesto. }\end{array}$ & $\begin{array}{l}\text { Disminución de } 7.6 \% \text { en compra de bebidas } \\
\text { azucaradas, con un impulso de menos de } 10 \% \text { del } \\
\text { valor y aumento en compra de agua y otras bebidas } \sin \\
\text { azúcar. }\end{array}$ \\
\hline Evaluación de efectos & $\begin{array}{l}\text { Evaluación de consumo de bebidas y } \\
\text { alimentos. } \\
\text { Evaluación de prevalencias de obesidad y } \\
\text { diabetes. }\end{array}$ & \\
\hline
\end{tabular}
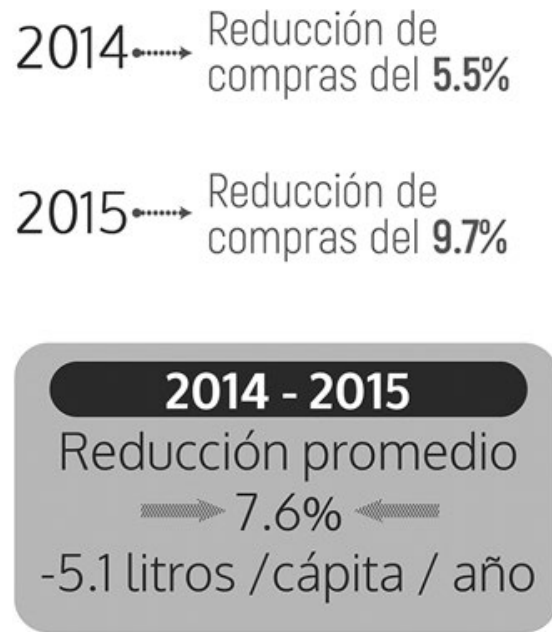

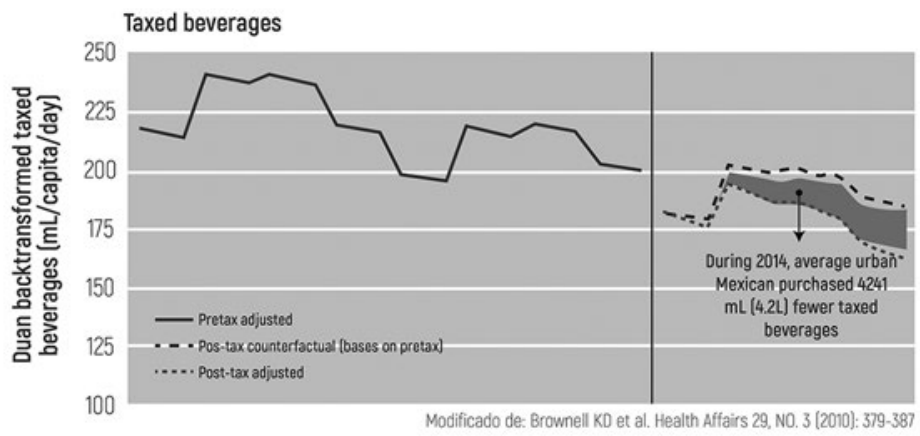

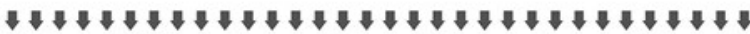
Reducciones sostenidas y crecientes

Figura 7. Efecto en la compra de bebidas azucaradas después del impuesto especial sobre productos y servicios. Fuente: Referencias ${ }^{51-53}$. A) Modificado de referencia 40.

En cuanto a las evaluaciones de implementación, si esta es acorde con las reglas los resultados serán positivos.

Respecto a las evaluaciones de resultado, un ejemplo sería la evaluación de la compra de bebidas y alimentos con o sin impuestos. El Instituto Nacional de Salud Pública tiene evidencia de que ha disminuido el consumo de bebidas azucaradas y ha aumentado el consumo de bebidas no azucaradas como resultado del IEPS (Figura 7)..$^{51-53}$

Finalmente, la evaluación de las prevalencias de obesidad y diabetes a través del consumo de alimentos y bebidas es un ejemplo de evaluación de efectos.

Aunado a lo anterior, es importante resaltar que todas las medidas regulatorias deben ser motivo de evaluación independiente.

\section{Conclusiones}

La prevención de las enfermedades crónicas no transmisibles requiere la adopción de conductas saludables como no consumir tabaco, llevar una dieta saludable, tener un consumo responsable de alcohol y realizar actividad física regular. Para adoptar estas conductas se requiere la creación de entornos saludables mediante la regulación, por lo que esta se vuelve indispensable para la prevención y control de enfermedades. El proceso regulatorio requiere evidencia científica para su diseño y evaluación rigurosa, a fin de lograr mayores efectos.

Finalmente, el proceso regulatorio requiere la rectoría del Estado, así como la participación de la academia, la sociedad civil y la industria conforme a reglas claras para evitar conflictos de interés. 


\section{El desarrollo de la capacidad para la regulación en salud en México}

Manuel Urbina-Fuentes

\section{Introducción}

La regulación para la equidad y el desarrollo sostenible en un mundo globalizado es uno de los retos en la adecuada gobernanza e implementación de políticas públicas. Son el resultado de la respuesta del Estado para garantizar el acceso, disponibilidad, calidad, asequibilidad, respeto, protección y promoción de los derechos humanos porque tienen una dimensión e impacto mundial y nacional. En la perspectiva del desarrollo de la capacidad de la regulación en salud para proteger a la población contra los riesgos y peligros sanitarios, la variable demográfica adquiere mayor importancia porque afecta las esferas económica, social y ambiental.

\section{Dimensión e impacto mundial}

El incremento acelerado de la población, principalmente en los países de bajo y medio desarrollo, ampliará los riesgos sanitarios por el impacto que ya ha tenido y porque se tendrán que enfrentar viejos y nuevos desafíos para un lograr un desarrollo sostenible con equidad. Somos 7500 millones de personas las que habitamos el mundo y México se encuentra en el décimo lugar entre los 20 países con mayor volumen de población. La población mundial crece a una tasa anual de aproximadamente $1.11 \%$, se estima un incremento de 80 millones por año y se llegará a los 10 mil millones en 2056 (seis años antes de lo estimado anteriormente). Actualmente, la mediana de edad es de 29.9 años, la tasa de fecundidad es de 2.5 hijos y $54.7 \%$ de la población es urbana con más de 4100 millones. ${ }^{54}$ Asimismo, la inequidad como fenómeno social, político y económico está empeorando y el crecimiento poblacional la magnificará.

A lo anterior se suman los diez riesgos globales que en el Foro Económico de Davos, Suiza, se han identificado en términos de probabilidad e impacto sobre la población mundial, agrupados en cinco categorías ampliamente descritas en el reporte de 2017:55

- Ambiental: falla en la control y adaptación del cambio climático y pérdida de la biodiversidad y colapso de ecosistemas.
- Geopolítica: armas de destrucción masiva.

- Social: crisis del agua, migración involuntaria masiva, propagación de enfermedades infecciosas, inestabilidad social profunda.

- Económica: crisis de precios para energía, crisis financieras y burbuja de activos financieros.

- Tecnológica: robo y fraude de datos.

Adicionalmente, en el ámbito del comercio, donde la regulación sanitaria es muy importante, implica conocer los escenarios mundiales de las 10 mayores economías del mundo. En 2017 dichas economías se valoraron en trillones de dólares: Estados Unidos con 18 (24.3\%), China con 11 (14.8\%), Japón con 4.4 (6\%), Alemania con 3.3, Reino Unido con 2.9, Francia con 2.4, India con 2.0, Italia con 1.8, Brasil con casi 1.8 y Canadá con más de 1.5. En un estudio de PricewaterhouseCoopers se pronostica que China estará en el primer lugar para 2050, India ocupará el segundo, Estados Unidos será tercer lugar y se espera que Indonesia, el cuarto. ${ }^{56}$

En todo el mundo, la regulación y el comercio han aumentado los ingresos y pueden reducir las inequidades, pero un estudio de 27 industrias y 13 países en desarrollo considera los riesgos actuales del comercio: con la salida del Reino Unido de la Unión Europea, la renegociación del Tratado de Libre Comercio entre Canadá, Estados Unidos y México y no lograr un acuerdo de asociación transpacífico entre Australia, Brunei Darussalam, Canadá, Chile, Estados Unidos, Japón, Malasia, México, Nueva Zelanda, Perú, Singapur y Vietnam se privaría al $10 \%$ más rico de $28 \%$ de su poder adquisitivo y el $10 \%$ más pobre perdería $63 \%$ porque compraría relativamente más bienes importados.

Un retroceso de la integración mundial erosionaría los logros, especialmente en los países en desarrollo. Según el Banco Mundial, el abandono de los acuerdos existentes en América Latina tendría efectos negativos en el bienestar de países como México (4 a $9 \%$ ), El Salvador (2 a $5 \%$ ) y Honduras (2 a $5 \%$ ). ${ }^{57}$

\section{Riesgos actuales y futuros del planeta}

Debido a que el crecimiento demográfico ha acontecido principalmente en los países en desarrollo se ha generado incertidumbre en la gestión de los 
recursos por el aumento en la demanda de agua, energía y alimentos, por lo que hay que considerar también que conllevan el desarrollo de la capacidad de la regulación, principalmente por los efectos en el cambio climático y los fenómenos meteorológicos. ${ }^{58}$ Ante estos hechos, diferentes investigadores y organizaciones especializadas han planteado que para contar con un espacio operativo seguro para la humanidad y un desarrollo económico inclusivo y sostenible no se deben rebasar nueve límites planetarios:

- Cambio climático.

- Uso del agua.

- Ciclos del nitrógeno y fósforo.

- Acidificación oceánica.

- Contaminación química.

- Carga atmosférica de aerosoles.

- Agotamiento del ozono.

- Pérdida de la biodiversidad.

- Cambio del uso de la tierra.

Los cuales se han denominado "techo ambiental o ecológico", por encima de los que se encuentra una degradación ambiental inaceptable. También se ha concluido que existe un soporte social por debajo del cual existe riesgo de una privación humana inaceptable de agua, ingresos, educación, resiliencia, trabajo, energía, igualdad social, equidad de género, salud y alimentos. ${ }^{59}$ Entre los límites del soporte social y el techo ambiental se encuentra un espacio seguro y justo para la humanidad. Si el desarrollo económico fuera inclusivo y sostenible, la humanidad prosperaría.

Sin embargo, se requiere un orden y regulación sanitaria en aspectos como la salud, el agua, la energía y los alimentos. Por ello, preocupa que cuatro de los nueve límites planetarios del techo ambiental estén seriamente afectados: cambio climático, ciclos de nitrógeno y fósforo, pérdida de biodiversidad y conversión de la tierra, y que estén por sumarse la contaminación del aire y la contaminación química. Hay un nuevo récord en el registro del dióxido de carbono $\left(\mathrm{CO}_{2}\right)$ en la atmósfera de la Tierra: se espera que se mantenga o suba y supere un nivel histórico de 400 partículas por millón. ${ }^{60}$ El $40 \%$ de la población mundial estará viviendo estrés hídrico severo en 2050, ya que al año se pierden 1 a 2.3 millones de hectáreas agrícolas por la degradación del suelo. Se han registrado 300000 muertes al año por el humo de los incendios y el mal uso de la tierra contribuye al aumento del paludismo. Otro riesgo es el consumo elevado de pescados y mariscos, que ha provocado $90 \%$ de sobreexplotación de las aguas marinas. De continuar afectando el ambiente, entre 2030 y 2050 se podrán perder 250000 vidas cada año por el cambio climático y sus efectos en la salud. ${ }^{61}$

El cambio climático y la contaminación del agua impactan la salud al impedir los servicios médicos por las intensas lluvias originadas de las tormentas tropicales o por los niveles de contaminación fluvial "fuera de la norma" con cobre y ácido sulfúrico derivados de la explotación minera.

\section{Referente internacional de la regulación en salud}

El Reglamento Sanitario Internacional (RSI) es un instrumento jurídico internacional que abarca medidas para prevenir la propagación transnacional de enfermedades infecciosas. Tiene como finalidad y alcance "prevenir, proteger, controlar y proporcionar una respuesta de salud pública a la propagación internacional de la enfermedad en formas que sean proporcionales y limitadas a los riesgos para la salud pública y que eviten interferencias innecesarias con el tráfico y el comercio internacionales". Hay cinco razones por las que el RSI es importante:

- Las amenazas para la salud no tienen límites.

- Los viajes y el comercio se hacen más seguros.

- Mejora de la seguridad sanitaria mundial.

- Las amenazas diarias se mantienen bajo control.

- Todos los sectores se benefician.

Se afirma que hasta que todos los sectores cumplan con el RSI, ningún país estará listo; son las variables demográfica y socioeconómica las que limitan su observancia. ${ }^{62}$ Los sistemas regulatorios no solo comprenden las reglas internacionales y nacionales, sino también las reglas desarrolladas por las instancias estatales de gobierno. La aplicación de una regulación supone el aumento del bienestar social o evitar su pérdida al corregir las fallas de mercado, bienes o servicios a la cual se dirige la acción gubernamental.

La importancia de la regulación se observa desde dos ángulos, uno teórico y otro práctico, porque la sociedad debe estar interesada en cómo se beneficia o afecta cuando no se tiene o no se cumple o evalúa su calidad.

\section{Derechos sociales y desarrollo de la capacidad para la regulación}

Garantizar el pleno ejercicio de los derechos sociales consagrados en la Constitución Política de los Estados Unidos Mexicanos, asegurando el acceso de toda la población al desarrollo social es el objetivo de la Ley General de Desarrollo Social, que en su artículo 


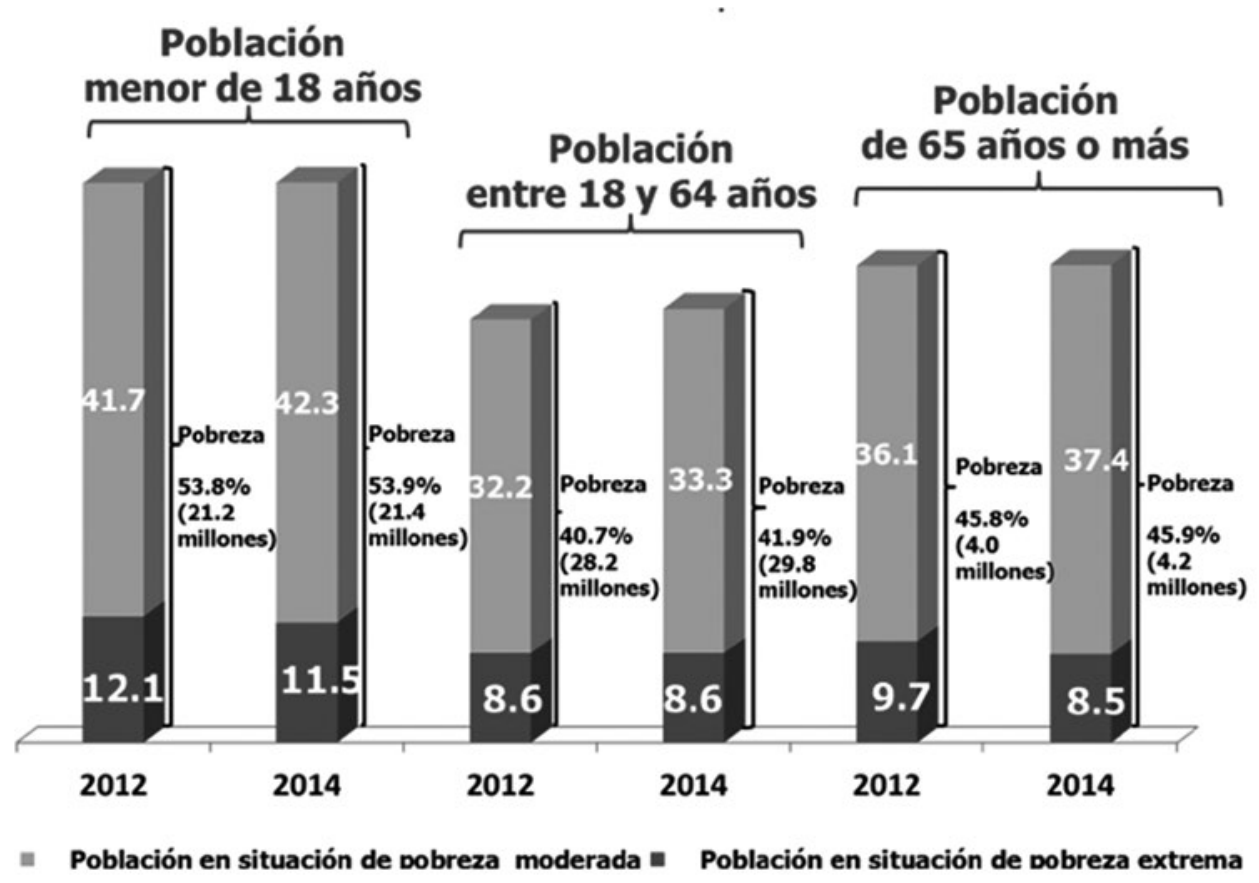

Figura 8. Porcentaje de personas en pobreza, según grupos de edad. México, 2012-2014.

6 establece los derechos sociales básicos de la población en México: educación, salud, alimentación nutritiva y de calidad, vivienda, disfrute de un medio ambiente sano, trabajo, seguridad social y no discriminación, derechos básicos para el desarrollo de la capacidad para la regulación porque identifican las zonas de atención prioritaria, con el propósito de dirigir las acciones más urgentes para superar las marcadas insuficiencias y rezagos en el ejercicio de los derechos para el desarrollo social, como la salud. ${ }^{63}$

La importancia de la regulación se destaca por el hecho de que entre 2005 y 2012, según la Comisión Nacional de los Derechos Humanos, en orden de importancia sobresalieron las quejas y demandas por violaciones a la salud, los derechos laborales y la educación, que se incrementaron de 3162 a 4616, de 0 a 2313 y de 868 a 1682, respectivamente. Asimismo, las quejas fueron en primer lugar en niños, niñas $y$ adolescentes, en segundo en migrantes y en tercer lugar en adultos mayores e indígenas. ${ }^{64}$

\section{Desigualdades, inequidad y exclusión}

Evaluar los logros o las injusticias sociales que disfruta o padece la población de un país, de un estado o de un municipio y sus localidades implica observar no solo sus condiciones de salud sino también la situación de las desigualdades, la inequidad y la exclusión en salud, es decir, las diferencias que se consideran indignas, remediables y evitables. Las clases sociales configuran no solo los resultados, sino las oportunidades de salud, ya que las sitúan en posiciones desiguales de poder, prestigio y acceso a los recursos y servicios. Las personas experimentan una exposición y vulnerabilidad diferencial ante factores que ponen en riesgo su salud como los determinantes sociales de la salud, que la Organización Mundial de la salud ha definido como "las condiciones en las que nacemos, crecemos, vivimos, educamos, trabajamos, divertimos, envejecemos y morimos". Son cuatro los entornos donde se dan principalmente las condiciones de las desigualdades o inequidades en salud: la vivienda, la escuela, el trabajo y los lugares donde nos recreamos o divertimos, que se manifestarán o magnificarán conforme a la posición socioeconómica, cultural y ambiental generales y su vinculación con las redes sociales y comunitarias a las que se pertenece, y a los factores y estilos de vida que se presentan según edad, sexo o carga genética de cada persona. ${ }^{65,66}$

Asimismo, la pobreza y la pobreza extrema son otros desafíos que afectan de manera creciente a la población del país y las opciones de acceso y beneficio de la regulación en salud. En México, el Consejo Nacional para la Evaluación de la Política Social en la medición que hace de la pobreza multidimensional de 2014 estimó que hay 55.3 millones de pobres (46.2\%) y que de estos, 11.4 millones son pobres extremos $(9.5 \%)$ (Figura 8). El porcentaje de personas en 
pobreza según grupos de edad es alarmante, en particular en los menores de 18 años (21.4 millones, $53.9 \%$ y los individuos de 65 años o más (4.2 millones, $45.9 \%$ ). ${ }^{67}$

En los 100 municipios con mayor marginación, predominantemente indígenas, un millón de mexicanos son los más pobres del país, la mayor parte en Chiapas $(30 \%)$, Oaxaca (20\%), Guerrero (17\%) y Veracruz (15\%). Adicionalmente, las niñas y los niños con riesgo de morir antes de cumplir un año son 1.7 veces mayor que el promedio nacional y la esperanza de vida de las mujeres alcanza 51 años y la de los hombres 49 años, aun cuando el promedio nacional es de 77.4 y 72.6 años, respectivamente. Las desigualdades de género son marcadas, 2.5 millones más de mujeres que hombres viven en condiciones de pobreza; hay 8 millones más de mujeres que hombres que trabajan y no cuentan con seguro médico ni derecho a una pensión. En el país, 6.9 \% de la población mayor de 15 años no sabe leer ni escribir: $5.6 \%$ de hombres y $8.1 \%$ de mujeres. El $32.5 \%$ de la población indígena mayor de 15 años es analfabeta y triplica al del hombre indígena ${ }^{68}$ Adicionalmente la educación primaria en México mantiene un retraso de 19 años en cobertura universal, de 44 años en secundaría y de 70 años en preparatoria, según el Informe de Seguimiento de la Educación en el Mundo 2016.

La regulación desempeña un papel importante para mejorar las condiciones, a pesar de lo cual $24.7 \%$ de las escuelas analizadas por el Instituto Nacional para la Evaluación de la Educación y el Fondo de las Naciones Unidas para la Infancia no tiene baño; solo en $59.7 \%$ de los casos, la lengua de los maestros coincide con la de los alumnos, $20.1 \%$ de las escuelas no tiene mobiliario para al menos un alumno y 49.9 $\%$ del total de los preescolares es atendido por un solo docente. ${ }^{69}$

\section{Salud sexual y reproductiva}

La salud sexual y reproductiva en México se ha afectado por el incremento de la fecundidad en los menores de 18 años. Varias causas han influido y condicionado esta realidad, cuyo mayor impacto es la mortalidad materna, los embarazos no deseados y el aborto. Las uniones tempranas, por ejemplo, relacionadas con la deserción escolar, el inicio temprano de la fecundidad, la pobreza y la violencia de género, reflejan que las niñas y los niños menores de 18 años, en la mayoría de los casos no tienen una mejor opción de vida, ya que sus padres los obligan a unirse.

Los matrimonios infantiles y las uniones tempranas ocurren por muchas razones: los individuos carecen de otras opciones de vida, se ven obligados a participar en arreglos maritales o piensan que es su mejor opción bajo las circunstancias y las normas sociales en las que viven. Sin embargo, son una violación a los derechos humanos, en particular de los niños. ${ }^{70}$ En mortalidad materna no se alcanzó la meta de los Objetivos del Milenio de 22.2 muertes por 100000 nacidos vivos para 2015, ya que se registraron 38.2. Tampoco se ha reducido la tasa de fecundidad adolescente en la última medición reportada en 2014: en lugar de disminuir se incrementó de 65.7 proyectada a 77 por $1000 . .^{71}$

Otro aspecto relevante son los embarazos no planeados, que en México ascienden a 71 por 1000 mujeres en edad reproductiva, de los cuales muchos terminan en abortos no inducidos (54\%), alrededor de un millón al año, a pesar de las restricciones legales en 31 entidades. Por ello, se debe destacar la incongruencia regulatoria que existe en la interrupción legal del embarazo no deseado, una opción accesible, disponible y segura que se tiene únicamente en la Ciudad de México desde 2007; el panorama para las mujeres en otros estados continúa siendo complicado debido a que no se tienen los servicios y, además, hay leyes que penalizan el aborto. Actualmente en 24 hospitales públicos de la Secretaría de Salud de la Ciudad de México se practican interrupciones legales del embarazo, a lo que se suman diversas clínicas privadas que prestan el servicio con seguridad. ${ }^{72}$ ¿Por qué no se ha regulado esta opción preventiva para reducir los riesgos en la salud de las mujeres? ${ }^{73}$

\section{Regulación en la adquisición y gasto de medicamentos}

El desabasto, surtimiento incompleto de recetas e incremento en los costos de medicamentos afectan las desigualdades sociales, no obstante que el porcentaje del gasto total en salud (28.3\%) y el porcentaje del producto interno bruto para medicamentos $(1.7 \%)$, de acuerdo con los indicadores de la Organización para la Cooperación y el Desarrollo Económicos, son muy superiores a los de los países miembros: 16.4 y $1.4 \%$, respectivamente (Figura 9). ${ }^{74}$ 


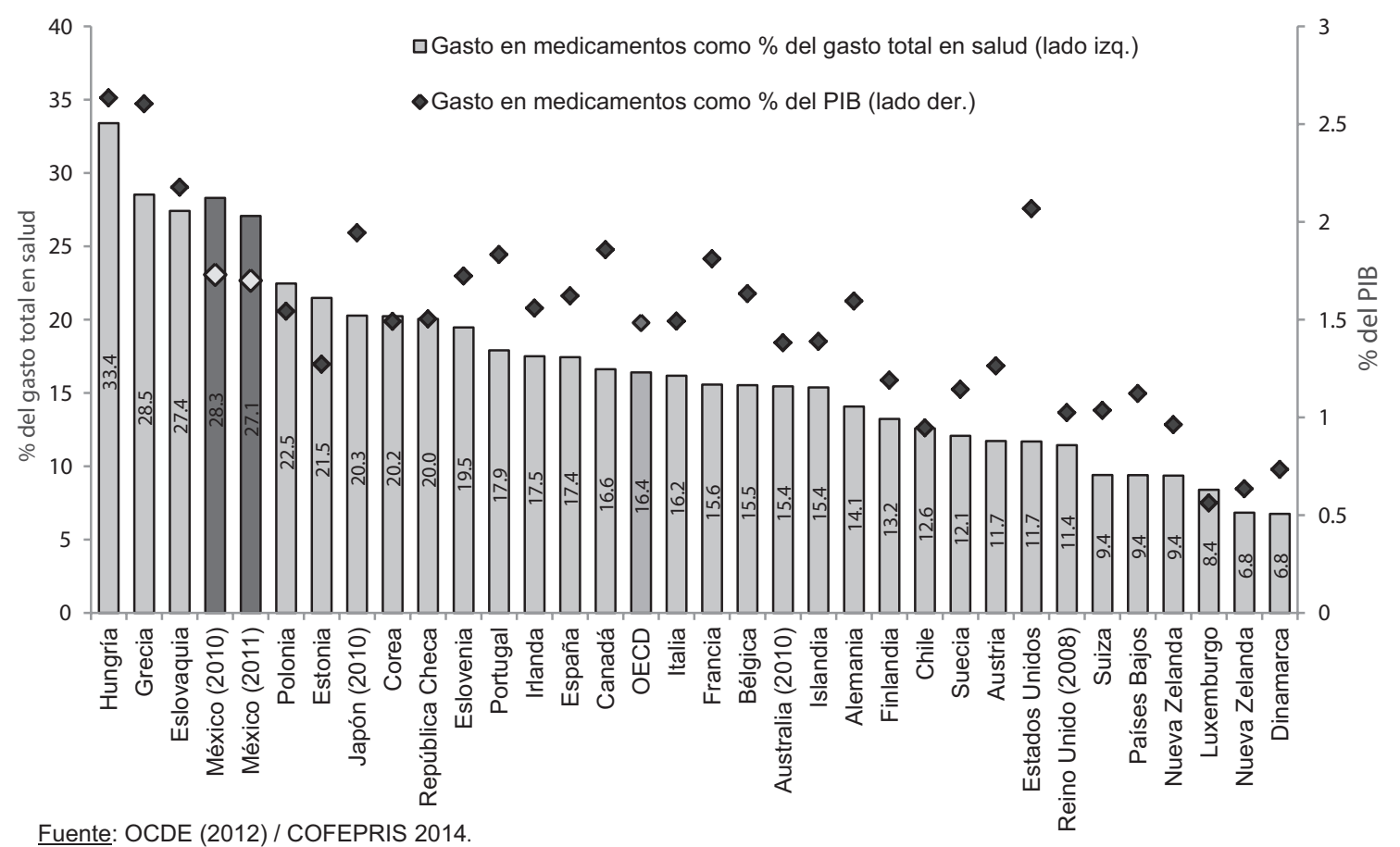

Figura 9. Gasto en medicamentos en diferentes países miembros de la Organización para la Cooperación y el Desarrollo Económicos.

\section{Agenda 2030: marco de la regularización para la equidad y el desarrollo sostenible}

La Agenda 2030 y los indicadores que comprometieron 193 países muestran la escala y la ambición de una nueva agenda universal, oportunidad para el desarrollo de la capacidad para la regulación en muchas dimensiones, especialmente en salud, alimentación, agua, vida submarina y vida de ecosistemas terrestres. Los 17 objetivos, las 169 metas y los 231 indicadores serán el marco de referencia para hacerlo, ya que para el 2030 se comprometió implementarlos a nivel nacional, regional y local. Se busca poner fin a la pobreza, reducir la desigualdad y luchar contra el cambio climático, garantizando que nadie se quede atrás en las dimensiones económica, social y ambiental..$^{75}$

\section{Papel social de la Academia Nacional de Medicina de México}

La Academia Nacional de Medicina de México debe analizar y promover las recomendaciones para la regulación en salud que se presentan y derivan de dos ámbitos complementarios y no excluyentes: los compromisos internacionales adquiridos por el gobierno de México y los normativos y programáticos nacionales para la regulación desde el marco de la evaluación del desempeño y del impacto de los componentes, bienes y servicios que otorga el sector salud. En los dos ámbitos deben considerarse las experiencias de éxito en otros países y las recomendaciones de los expertos nacionales e internacionales. 


\title{
Comentario sobre el desarrollo de la capacidad para la regulación en salud en México
}

\author{
Fernando Cano-Valle \\ Academia Nacional de Medicina, Ciudad de México, México
}

\begin{abstract}
Un importante componente de la salud es la prevención. El ambiente físico y social impacta en la salud de la población. La Comisión Federal para la Protección contra Riesgos Sanitarios (Cofepris) es el organismo de la Secretaría de Salud encargado de vigilar las actividades que impactan la salud. La Cofepris tiene el encargo de proteger a la población de los eventos exógenos que ponen en riesgo la salud o la vida humana. Estos eventos pueden ser la exposición a factores biológicos, químicos o físicos presentes en el medio amiente o en los productos o servicios que se consumen. ${ }^{1}$
\end{abstract}

Con base en un estudio realizado durante dos años en relación con diversas instancias de salud, considero que la naturaleza jurídica de la Cofepris entra en duda cuando analizamos en conjunto su diseño institucional, porque no parece un órgano desconcentrado de la Secretaría de Salud sino un descentralizado. ${ }^{76}$ Se fractura del principio de especialización constitucional, plasmado en la base $2^{a}$. de la fracción XVI del artículo 73 de la Constitución Política de los Estados Unidos Mexicanos, porque quien debe ejercer las competencias en materia de riesgo sanitario es el secretario de salud, no un comisionado federal.

El Plan de Acción de la Cofepris destaca que riesgo es un evento exógeno que pone en peligro la salud o la vida humana como resultado de la exposición, generalmente involuntaria, a factores biológicos químicos o físicos presentes en el medio ambiente, o por el consumo de productos y servicios, incluyendo la publicidad. ${ }^{77}$

El riesgo sanitario es resultante de la probabilidad de ocurrencia de un evento adverso conocido o potencial a la salud y su severidad asociada a factores o elementos biológicos, químicos y físicos por consumo o uso de agua, alimentos, bebidas, medicamentos, equipos médicos, productos de perfumería, belleza y aseo, nutrientes vegetales, plaguicidas, sustancias tóxicas o peligrosas y otros productos, sustancias o agentes físicos, químicos o biológicos presentes en el medio ambiente o en el trabajo, así como mensajes publicitarios de productos y servicios. ${ }^{77}$

Un riesgo sanitario es un factor, detectable, medible e identificable, que se sabe o se intuye puede causar una condición desfavorable en la salud de la población, que no se daría sin la presencia de ese factor. ${ }^{77}$

\section{Equidad}

Para George Alleyne -enviado especial de las Naciones Unidas para el VIH/SIDA en la región del Caribe 2003-2010; nombrado por Kofi Annan, Secretario General de las Naciones Unidas, en febrero de 2003-, la equidad en materia de salud es fundamentalmente, es un tema ético-moral y no debemos negarnos a considerarlo tal. Se acepta que la salud está condicionada por características biológicas, pero que esencialmente es un concepto social. Las desigualdades de salud que tienen origen social son injustas e injustificadas por que representan la distribución de los recursos de la sociedad, de tal manera que ciertos grupos no disponen de oportunidades o de libertades humanas básicas. Los sistemas fragmentados de salud, con su acceso compartimentalizado ligado a las fuentes y mecanismos de financiamiento, han creado una segmentación desigual de la población en lo concerniente a la cobertura por los servicios de salud. Además, la magnitud de los gastos directos de bolsillo en salud, así como las pronunciadas inequidades en la distribución de la carga financiera entre los distintos quintiles de la población producen brechas aún mayores en el acceso a la provisión de servicios de atención a la salud.

Al cabo del tiempo, quizás el impacto negativo más significante está en relación con la inequidad; si se acepta el criterio de que la pobreza es una privación de ciertas capacidades, una de las cuales es la salud, entonces, por definición, todo lo que se haga por mejorar la salud reduce la pobreza. Conforme los conceptos de George Alleyne, en México el progreso se ha visto obstaculizado por una serie de factores entre los que destacan:

- La falta de voluntad política.

- La falta de equidad en el acceso a todos los elementos de atención primaria de salud.

- La persistente baja consideración de las mujeres.

- El desarrollo económico lento.

- La dificultad para conseguir una acción intersectorial en materia de salud. 
- La distribución desequilibrada de los recursos humanos y el escaso apoyo a los mismos.

- La inadecuación generalizada de las actividades de promoción de la salud.

- Los sistemas deficientes de información sanitaria.

- La contaminación, la insuficiente inocuidad de los alimentos y la falta de suministros de agua salubre y de saneamiento.

- Los rápidos cambios demográficos y epidemiológicos.

- La inadecuada asignación y utilización de los recursos para tecnologías costosas.

- Los desastres naturales y producidos por el hombre.

La atención efectiva a los derechos humanos implica políticas públicas que dispongan de una fuente presupuestal de ingresos fiscales recurrentes para cubrir los costos, o bien, una hacienda pública con buenas intenciones de acuerdo con las necesidades de la sociedad mexicana.

No sorprende que en la actualidad, la fragmentación del sistema público de la salud implique la duplicación de las funciones, la cual, estrechamente relacionada con la inexistencia de un sistema nacional confiable de información, desaprovecha la planeación estratégica de los recursos para la salud.

Es necesario repensar el propósito de las políticas públicas en salud a la luz y necesidad imperiosa de los derechos humanos. La sinergia virtuosa de los derechos humanos representa la semilla del análisis moderno de las políticas públicas en salud, cuyas respuestas hasta el momento han sido insuficientes. ${ }^{78}$

La reforma o los cambios de mayor peso y trascendencia para la protección del ciudadano que puede verse afectado ante la inconsistencia de una política de salud presupone la existencia de un Estado con capacidad efectiva para ejercer el orden jurídico que garantice el desempeño de su función. No hay igualdad legal posible ante programas o proyectos limitadamente desarrollados.

En un marco de inequidad que repercute en la sociedad...

Los problemas en materia de regulación de la adicción de harinas y azúcares, sus vínculos con la obesidad infantil, "productos milagro" y el tema de las competencias en materia laboral, muestran una serie de disfunciones en el operador en materia de riesgo sanitario, que requieren atención, pero, sobre todo, nos llevan a la necesidad de mostrar hasta dónde se deben a problemas de diseño normativo, a deficientes estructuras administrativas o a ineficiencia del aplicador del derecho en el nivel administrativo. ${ }^{78}$

\section{Bibliografía}

1. Howard PC, Tong W, Weichold F, Healy M, Slikker W. Global summit on regulatory science 2013. Regul Toxicol Pharmacol. 2014;70:728-732.

2. Organización Panamericana de la Salud. Función rectora de la autoridad sanitaria. Marco conceptual/instrumento metodológico. EE. UU.: OPS; 2007.

3. Bettcher DW, Sapirie S, Goon EH. Essential public health functions: results of the international Delphi Study. World Health Stat Q. 1998; 51(1):44-54.

4. Knaul FM, González-Pier E, Gómez-Dantés O, García-Junco D, Arreola-Ornelas $H$, Barraza-Lloréns $M$, et al. The quest for universal health coverage: achieving social protection for all in Mexico. Salud Publica Mex. 2013:55:207-235.

5. Fine P, Victora CG, Rothman KJ, Moore PS, Chang Y, Curtis V, et al. Snow's legacy: epidemiology without borders. Lancet. 2013;381: 1302-1311.

6. Romero-Álvarez H. Semblanza del Dr. Bustamante. Salud Publica Mex. 1975;17(5):719-724.

7. Sen A. Development as freedom. EE. UU.: Oxford University Press; 2001.

8. North DC. Understanding the process of economic change. EE.UU.: Princeton University Press; 2005.

9. Tirole J. Market failures and public policy. Am Econom Rev. 2015, 105:1665-1682.

10. Stigler GJ. The theory of economic regulation. Bell J Econ Manag Sci. 1971;2:3-21.

11. Organización Panamericana de la Salud. Factores de riesgo de las enfermedades no transmisibles en la región de las Américas: consideraciones para fortalecer la capacidad regulatoria. Documento técnico de referencia REGULA. EE. UU.: OPS/OMS; 2016

12. Verguet S, Kim JJ, Jamison DT. Extended cost-effectiveness analysis for health policy assessment: a tutorial. Pharmacoeconomics. 2016;34:913-923.

13. Peña CE, Carter D, Ayala-Fierro F. Toxicología ambiental. Evaluación de riesgos y restauración ambiental. EE.UU.: The University of Arizona; 2001.

14. Miller W, Robinson LA, Lawrence RS, editores. Valuing health for regulatory cost-effectiveness analysis. EE. UU.: Institute of Medicine; 2006.

15. Murphy K. Walt Whitman and Louis Sullivan: the aesthetics of egalitarianism. WWQR. 1988;6:1-15.

16. International Risk Governance Council. Introduction to Risk Governance. Ginebra, Suiza: IRGC; 2012. Disponible en: https://www.irgc.org/risk-governance/irgc-risk-governance-framework.

17. Decreto por el que se crea la Comisión Federal para la Protección contra Riesgos Sanitarios. México: Diario Oficial de la Federación; 2001 Jul 5.

18. Secretaría de Salud. Reglamento de la Comisión Federal de Protección contra Riesgos Sanitarios. México: Diario Oficial de la Federación 2004 Abr 13.

19. Hausman R, Hidalgo CA, Bustos S, et al. The atlas of economic complexity. Mapping path to prosperity. EE. UU.: Center for International Development; 2011.

20. Labonté R, Schram A, Ruckert A. The trans-pacific partnership: Is it everything we feared for health? Int J Health Policy Manag. 2016;5:487-496.

21. Ley General de Salud. Disponible en: http://www.cofepris.gob.mx/MJ/ Paginas/Reglamentos.aspx.

22. Comisión Federal para la Protección contra Riesgos Sanitarios. Farmacopea de los Estados Unidos Mexicanos. México: Secretaría de Salud; 2009.

23. Comisión Federal para la Protección contra Riesgos Sanitarios. Reglamento de Insumos para la Salud. Diario Oficial de la Federación 2014 Mar 14. Disponible en: http://www.cofepris.gob.mx/MJ/Paginas/ Reglamentos.aspx

24. Comisión Federal para la Protección contra Riesgos Sanitarios. Gestión de la salud pública en México. México. Cofepris; 2015. Disponible en: http://www.cofepris.gob.mx/Documents/NotasPrincipales/08012015.pdf

25. Secretaría de Salud. Informe sobre la seguridad vial, México 2015. México: Secretaría de Salud; 2016.

26. Gómez-Dantes H, Fullman N, Lamadrid-Figueroa H, Cahuana-Hurtado L, Darney B, Ávila-Burgos L, et al. Dissonant health transition in the states of Mexico, 1990-2013: a systematic analysis for the Global Burden of Disease Study 2013. Lancet. 2016;388:2386-2402.

27. Portier CJ. Report of PAHO/WHO Collaborating Centers Meeting. EE. UU.: 2011.

28. Peden MM, Scurfield R, Mohan D, Hyder AA, Jarawan E, Mathers C, editores. World report on road traffic injury prevention. Ginebra, Suiza: WHO; 2004.

29. United Nations General Assembly. Resolution adopted by the General Assembly: 64/255. Improving global road safety. EE. UU.: UN; 2010.

30. Acuerdo por el que se da a conocer la Estrategia Nacional de Seguridad Vial 2011-2020. México: Diario Oficial de la Federación 2011 Jun 6. Disponible en: http://dof.gob.mx/nota_detalle.php\%3Fcodigo\%3D5193284\%26fecha\%3D06/06/2011 
31. Decreto por el que se crea el Consejo Nacional para la Prevención de Accidentes. México: Diario Oficial de la Federación 1987 Mar 20. Disponible en: http://www.salud.gob.mx/unidades/cdi/nom/compi/d200387.html

32. Secretaría de Salud. Programa de Acción Específico. Seguridad Vial 2013-2018. México: Secretaría de Salud; 2014.

33. Secretaría de Economía, Secretaría de Salud. Proyecto de norma oficia mexicana PROY-NOM-206-SCFI/SSA2-2016. Cascos de seguridad para la prevención y atención inmediata de lesiones en la cabeza de motociclistas-Acciones de promoción de la salud-especificaciones de seguridad y métodos de prueba, información comercial y etiquetado. México: Diario Oficial de la Federación 2017 Abr 17. Disponible en: http://www.dof.gob.mx/nota_detalle.php?codigo=5479920\&fecha $=17 / 04 / 2017$

34. Constitución Política de los Estados Unidos Mexicanos, articulo 115 fracción III inciso h) disposición por virtud del cual se confiere a favor de los municipios, entre otros, las funciones y servicios públicos de tránsito. Diario Oficial de la Federación 1993 Dic 23

35. Hijar M, editora. Los accidentes como problema de salud pública en México: retos y oportunidades. Academia Nacional de Medicina/Consejo Nacional de Ciencia y Tecnología; 2014.

36. Barss $P$, Smith G, Baker S. Mohan D. Injury prevention an international perspective epidemiology, surveillance and policy. EE. UU.: Oxford University Press; 1998

37. Organización Mundial de la Salud. Enfermedades no transmisibles 2017. [Citedo 2017 Jun 10]. Disponible en: http://www.who.int/mediacentre/ factsheets/fs355/es

38. Hernández-Cordero S, Cuevas-Nasu L, Morán-Ruán MC, Méndez-Gómez Humaran I, Ávila-Arcos MA, et al. Overweight and obesity in Mexican children and adolescents during the last 25 years. Nutr Diabetes. 2017;7 e247.

39. Barquera S, Campos-Nonato I, Hernández-Barrera L, Pedroza A, Rivera-Dommarco JA. Prevalence of obesity in Mexican adults 2000-2012. Salud Publica Mex. 2013;55:S151-S160.

40. Brownell KD, Kersh R, Ludwig DS, Post RC, Puhl RM, Schwartz MB et al. Personal responsibility and obesity: a constructive approach to a controversial issue. Health Aff (Millwood). 2010;29:379-387.

41. Huang TT, Cawley JH, Ashe M, Costa SA, Frerichs LM, Zwicker L, et al. Mobilisation of public support for policy actions to prevent obesity. Lancet. 2015;385:2422-2431.

42. Safdie $M$, Jennings-Aburto $N$, Lévesque $L$, Janssen I, Campirano-Núñez $\mathrm{F}$, López-Olmedo $\mathrm{N}$, et al. Impact of a school-based intervention program on obesity risk factors in Mexican children. Salud Publica Mex. 2013:55:374-387.

43. Théodore FL, Tolentino-Mayo L, Hernández-Zenil E, Bahena L, Velasco A, Popkin B, Rivera JA, Barquera S. Pitfalls of the self-regulation of advertisements directed at children on Mexican television. Pediatr Obes. 2017:12:312-319.

44. Te Morenga L, Mallard S, Mann J. Dietary sugars and body weight: systematic review and meta-analyses of randomised controlled trials and cohort studies. BMJ. 2012;346:e7492.

45. Batis C, Aburto TC, Sanchez-Pimienta TG, Pedraza LS, Rivera JA. Adherence to dietary recommendations for food group intakes is low in the Mexican population. J Nutr. 2016;146(9):1897S-906S.

46. Colchero MA, Zavala JA, Batis C, Shamah-Levy T, Rivera-Dommarco JA Changes in prices of taxed sugar-sweetened beverages and nonessential energy dense food in rural and semi-rural areas in Mexico. Salud Publica Mex. 2017:59:137-146.

47. Sánchez-Pimienta TG, Batis C, Lutter CK, Rivera JA. Sugar-sweetened beverages are the main sources of added sugar intake in the Mexican population. J Nutr. 2016:146:1888S-1896S.

48. Colchero MA, Salgado JC, Unar-Munguia M, Hernández-Ávila M, Rivera-Dommarco JA. Price elasticity of the demand for sugar sweetened beverages and soft drinks in Mexico. Econ Hum Biol. 2015;19:129-137.

49. Rincón-Gallardo Patiño S, Tolentino-Mayo L, Flores Monterrubio EA Harris JL, Vandevijvere S, Rivera JA, Barquera S. Nutritional quality of foods and non-alcoholic beverages advertised on Mexican television according to three nutrient profile models. BMC Public Health. 2016;16: 733.

50. Arrúa A, Curutchet MR, Rey N, Barreto P, Golovchenko N, Sellanes A, et al. Impact of front-of-pack nutrition information and design on children's choice of two snack foods: Comparison of warnings and the traffic-light system. Appetite. 2017;116:139-146.

51. Colchero MA, Guerrero-López CM, Molina M, Rivera JA. Beverages sales in Mexico before and after implementation of a sugar sweetened beverage tax. PLoS One. 2016;11:e0163463.

52. Colchero MA, Ng SW, Popkin BM. Sugar-sweetened beverage tax: The authors reply. Health Aff (Millwood). 2017;36:1145.
53. Colchero MA, Popkin BM, Rivera JA, Ng SW. Beverage purchases from stores in Mexico under the excise tax on sugar sweetened beverages: Observational study. BMJ. 2016;352:h6704.

54. United Nations. World population prospects: The 2015 revision. Methodology of the United Nations population estimates and projections. EE. UU.: UN; 2015

55. World Economic Forum. The Global Risks Report 2017. Décima segunda edición. Ginebra, Suiza: World Economic Forum; 2017.

56. Hawksworth J, Clarry R, Audino $\mathrm{H}$. The Long view. How will the global economic order change by 2050? Reino Unido: PricewaterhouseCoopers; 2017.

57. World Bank Group. Global economic prospects. January 2017. Weak investment in uncertain times. EE. UU.: World Bank Group; 2017.

58. Rockström J, Steffen W, Noone K, Persson A, Chapin FS $3^{\text {rd }}$, Lambin EF, et al. A safe operating space for humanity. Nature. 2009;461:472-475.

59. Raworth KA. Doughnut for the Anthropocene: Humanity's compass in the $21^{\text {st }}$ Century. The Lancet-Planetary Health. 2017;1:e48-e49.

60. National Oceanic and Atmospheric Administration. [Sitio web]. Stein T. Record annual increase of carbon dioxide observed at Mauna Loa for 2015. Carbon dioxide at NOAA's Mauna Loa Observatory reaches new milestone: tops 400 ppm. National Oceanic and Atmospheric Administration. [Actualizado 2016 Mar 9]. Disponible en: http://www.noaa.gov/news/record-annualincrease-of-carbon-dioxide-observed-at-mauna-loa-for-2015

61. Whitmee S, Haines A, Beyrer C, Boltz F, Capon AG, Ezeh A, et al. Safeguarding human health in the Anthropocene Epoch: Report of The Rockefeller Foundation-Lancet Commission on planetary health. Lancet. 2015;386:1973-2028.

62. Organización Mundial de la Salud. Consulta regional sobre el marco de monitoreo del Reglamento Sanitario Internacional después del 2016. CD54/INF/4, Add. 20 de agosto del 2015. 54 Consejo Directivo. 67 sesión del Comité Regional de la OMS para las Américas, Washington DC, del 28 de septiembre al 2 de octubre del 2015.

63. Ley General de Desarrollo Social. México: Diario Oficial de la Federación 2018 Ene 26. Disponible en: http://www.diputados.gob.mx/LeyesBiblio/ pdf/264_260118.pdf

64. Programa Nacional de Derechos Humanos 2014-2018. México: Diario Oficial de la Federación 2014 Abr 30. Disponible en: http://www.dof.gob. $\mathrm{mx} /$ nota_detalle.php?codigo $=5343071 \&$ fecha $=30 / 04 / 2014$

65. World Health Organization. A conceptual framework for action on the social determinants of health. Ginebra, Suiza: WHO; 2007.

66. Organización Mundial de la Salud. $62^{2}$ Asamblea Mundial de la Salud. Ginebra: OMS; 2009.

67. Consejo Nacional de Evaluación de la Política de Desarrollo Social. Informe de pobreza en México 2014. México: Coneval; 2016.

68. Consejo Nacional de Evaluación de la Política de Desarrollo Social. Diagnóstico de los objetivos e indicadores de los planes derivados del Plan Nacional de Desarrollo 2013-2018. México: Coneval; 2015.

68. Organización de las Naciones Unidas para la Educación la Ciencia y la Cultura. Informe de Seguimiento de la Educación en el Mundo 2016. La educación al servicio de los pueblos y el planeta: creación de futuros sostenibles para todos. Francia: UNESCO; 2017.

70. Palma JL, Rivero E. Informe sobre uniones tempranas en México. Resumen ejecutivo. México: Investigación en Salud y Demografía; 2017.

71. Oficina de la Presidencia de la República. Objetivos de Desarrollo del Milenio en México 2015. Informe de avances. México: Presidencia de la República; 2015.

72. Instituto Nacional de Estadística y Geografía. Encuesta Nacional de la Dinámica Demográfica 2014. México: INEGI; 2015.

73. Juárez F, Singh S, Maddow-Zimet I, Wulf D. Embarazo no planeado y aborto inducido en México: causas y consecuencias. EE. UU.: Guttmacher Institute; 2013.

74. Comisión Federal para la Protección contra Riesgos Sanitarios. Gestión de la Salud Pública en México: Regulación sanitaria. México: Cofepris; 2014.

75. United Nations. Transforming our world: The 2030 Agenda for sustainable development. EE. UU.: UN; 2015.

76. Cano-Valle F, Márquez-Gómez D, Melgar-Manzanilla P. Medicina y estructuras jurídico-administrativas en México. Hacia la reforma integral del sistema de salud mexicano. México: Universidad Nacional Autónoma de México/Instituto de Investigaciones Jurídicas; 2014.

77. Cano-Valle F, Márquez-Gómez D, Melgar-Manzanilla P. Medicina y estructuras jurídico-administrativas en México. Hacia la reforma integral del sistema de salud mexicano. México: Universidad Nacional Autónoma de México/Instituto de Investigaciones Jurídicas; 2014.

78. Cano-Valle $F$, Pantoja-Nieves $M$, Sánchez-Miguel M. El péndulo de la medicina. El doble péndulo de la salud urge una reforma en el sistema de salud. México: Editorial Prado/Universidad Nacional Autónoma de México/Instituto de Investigaciones Jurídicas; 2017. 